\title{
Back Side Illuminated scientific CMOS detector for soft X-ray Resonant Scattering and Ptychography
}

Kewin Desjardins ${ }^{a}$, Kadda Medjoubi ${ }^{a}$, Pascal Mercère ${ }^{a}$, Paulo Da Silva ${ }^{a}$, Horia Popescu $^{a}$, Roland Gaudemer ${ }^{a}$, M. Sacchi ${ }^{a}$, Adrien Besson ${ }^{a}$, Rachid Belkhou ${ }^{a}$, Stefan Stanescu $^{a}$, Sufal Swaraj ${ }^{a}$, Stéphanie Pautard ${ }^{a}$, Arafat Noureddine ${ }^{a}$, Fabienne Orsini ${ }^{a}$ and Nicolas Jaouen ${ }^{\mathrm{a}}$

${ }^{a}$ Synchrotron SOLEIL, Saint-Aubin, Gif-sur-Yvette, 91192, France

Correspondance email: kewin.desjardins@ synchrotron-soleil.fr

Abstract Huge progress have been done with the $3^{\text {rd }}$ generation storage ring, and more recently the ultimate storage ring under development suggest an unprecedent increase of $\mathrm{x}$-ray brightness. Unfortunately, as far as the detectors are concerned, progress has not been as fast and even more so in the range of soft $\mathrm{x}$-rays. In particular for 2D detector the most commonly used detector are based on old CCD technology and the recent development of CMOS detector will be certainly crucial for 2D detector in the soft x-rays. With this goal we explore the possibilities and the the performance of a camera equipped with new mass-marketed scientific Complementary Metal Oxide Semiconductor Back Side Illuminated (sCMOS-BSI) integrated in vacuum environment for soft X-ray experiment at synchrotron. The $4 \mathrm{Mpix}$ sensor reaches a frame rate up to $48 \mathrm{fps}$ while suiting the necessary performances needed for X-ray experiments, in term of linearity (98\%), homogeneity (PRNU <1\%) charge capacity (up to $80 \mathrm{ke}-$ ), readout noise (down to $2 \mathrm{e}-\mathrm{rms}$ ) and adequate dark current ( $3 \mathrm{e}-/ \mathrm{s} / \mathrm{px}$ ). The sensor performances tests in the X-ray range have been performed at the SOLEIL METROLOGIE beamline. The Quantum Efficiency, the spatial resolution $(24 \mathrm{lp} / \mathrm{mm})$, the energy resolution $(<100 \mathrm{eV})$ and the radiation damage versus the $\mathrm{X}$-ray dose $(<200 \mathrm{~Gy})$ have been evaluated in the energy range from $40 \mathrm{eV}$ to $2000 \mathrm{eV}$. In order to illustrate the capabilities of this new sCMOSBSI sensor, several experiments have been performed at three soft x-ray beamline of the SOLEIL synchrotron: diffraction pattern from a pinhole at $186 \mathrm{eV}$, scattering experiment from nanostructured $\mathrm{Co} / \mathrm{Cu}$ multilayer at $700 \mathrm{eV}$ and Ptychoraphy imaging in transmission at $706 \mathrm{eV}$.

X-ray detectors, sCMOS camera, 2D Soft X-ray detector, soft X-ray scattering, ptychography, Back illuminated CMOS, Quantum efficiency, radiation hardness 


\section{Introduction}

In the last generation of synchrotron sources, the beamlines flux and beam coherence have been largely improved allowing to develop a large panel of sophisticated synchrotron radiation experiments. Nowadays, a lot of direct highly efficient detectors have been developed and are commercialized for hard X-ray application (Förster et al., 2019). They have demonstrated their high frame rates, high charge capacity, high efficiency and required high spatial resolution. However, a wide gap exists in the soft to tender X-ray domain (10 eV to $3 \mathrm{keV}$ ) where the experiments are not able to fully benefit from the performances offered by modern facilities. The detectors usually installed on the soft x-ray end-station are widely based on back-side illuminated CCD sensor such as the commercial cameras from Princeton@ PI-MTE (https://www.princetoninstruments.com), GreatEye® GE-VAC (https://www.greateyes.de) or others equipped with cooled CCD-BSI. This kind of detector is compact enough, vacuum compatible $\left(10^{-9} \mathrm{mbar}\right)$, presents performances largely demonstrated on laser based x-ray sources (Lambert et al, 2015), XFEL facilities (Pedersoli et al., 2011) or synchrotron beamlines (Chauleau et al, 2018). But these sensors suffers from severe readout speed limitation. Indeed, in order to limit the readout noise and so to guarantee a high signal to noise ratio allowing single photon sensitivity, the frame rate has to be limited to less than 1 frame per second. As an example, on SEXTANTS (Sacchi et al., 2013), the synchrotron SOLEIL's soft x-ray scattering beamline, they report a deadtime of $97.5 \%$, due to the readout time in full-chip mode (typically $4 \mathrm{~s}$ over $0.1 \mathrm{~s}$ of X-ray collection), during Xray Fourier Transform Holography (FTH) experiments on the COMET end-station (Popescu et al, 2018). Nevertheless, some solutions have been implemented to tackle this problem: (i) The fast-CCD (Denes et al., 2009) performs low readout noise with high frame rate (60 fps for $960 \times 1920$ pixels) by increasing the readout ports (ii) Other efforts have been done on Hybrid Pixel detectors (Jungmann-Smith et al., 2016, Wernecke et al. 2013) by decreasing the electronic noise level and optimizing the sensor entrance window allowing to profit of very high frame rate of new ASIC. (iii) Spectroscopic detector dedicated as pnCCD [Strüder 2016] could also be cited. (iv) the development of CMOS based sensor such Silicon-OnInsulator (SOI) technology (Arai et al, 2010) or DEPFET sensors (Porro et al, 2012) for very high frame rate soft X-ray FEL applications, the CMOS PERCIVAL detector (Wunderer et al., 2014) again under development by a consortium of synchrotrons or also some developments teams effort as the Pipper MAPS technologies recently published (El Bitar 2019). 
Recently, a new generation of large Back-Side Illuminated scientific CMOS sensor (sCMOSBSI) has been developed (Ma et al.,2015) and supplied by GPIXEL® (https://gpixel.com). Especially designed in order to optimize the efficiency delivered in the visible light range (> 95\%@550 $\mathrm{nm}$ ), and keeping the good performances of the first generation of sCMOS (low readout noise, large full well capacity and high frame rate), this new cost efficient $4 \mathrm{Mpx}$ sensor appears to be well suited for the soft x-ray applications, and has been already characterized for several use as soft X-ray resonant scattering (Desjardins et al., 2019) or Xray imaging spectroscopy (Wang et al., 2019). In this paper, we describe the integration of this sCMOS-BSI into an in-house vacuum compatible camera and its characterization. The performances have been measured on the METROLOGIE beamline (Idir et al, 2006) and are reported in the following sections. In particular, we have characterized the sCMOS-BSI camera dynamics (dark noise, readout noise, spatial uniformity, linearity and overall gain), the soft X-ray Quantum Efficiency in the energy range between 30 to $2000 \mathrm{eV}$, as well as the spatial, energy resolution and the radiation tolerance. We also report on different tests using this sCMOS-BSI camera: coherent diffraction pattern from a $5 \mu \mathrm{m}$ pinhole at $186 \mathrm{eV}$ at the METROLOGIE beamline, a soft X-ray Resonant Scattering experiment from nanostructure $\mathrm{Co} / \mathrm{Cu}$ multilayer at $700 \mathrm{eV}$ on SEXTANTS beamline and several soft X-ray ptychography reconstruction also at $706 \mathrm{eV}$ on HERMES beamline (Belkhou et al, 2015).

\section{Materiel}

\section{1. sCMOS GSENSE400BSI characteristics (Signal to Noise ratio in X-ray domain)}

The integrated and characterized sensor is the GSENSE400BSI (figure 1a), a scientific CMOS Back Side Illuminated from GPIXEL® (https://gpixel.com). This sCMOS-BSI sensor is a 4 megapixels ( $2048 \times 2048$ resolution, $11 \mu \mathrm{m}^{2}$ pixel size) high dynamic range sensor based on standard 4T pixel architecture (Ma et al.,2015) with two electronics gains: High Gain (HG), Low Gain (LG) and a computing combined gain mode in order to achieve the socalled HDR (High Dynamic Range) mode. Firstly integrated by TUCSEN® (http://www.tucsen.com) on Dhyana95 camera, this sensor in now largely integrated as on the PHOTOMETRICS ${ }^{\circledR}$ Prime95 (www.photometrics.com), the ANDOR ${ }^{\circledR}$ Sona (https://andor.oxinst.com) or others. These cameras take advantage of the sensor characteristic in term of readout noise $(<2$ e- rms), relatively large Full Well Capacity (FWC up to $80 \mathrm{ke}-$ ), good performances in term of spatial noise (DSNU $<5$ e- and PRNU $<1 \%$ ) 
and respectable result in term of dark noise (> $3 \mathrm{e}-/ \mathrm{s} /$ pixel with sensor cooled at $-20^{\circ} \mathrm{C}$ ). The full frame GSENSE400BSI sensor acquisition speed rises up to $48 \mathrm{~Hz}$ for standard gain mode (LG \& HG) and is limited to $24 \mathrm{~Hz}$ for the HDR mode. These performances, summarized in table 1, have been experimentally validated in visible with the Dhyana95 camera (Desjardins 2019, Wang 2017) following the EMVA normed (https://www.emva.org).

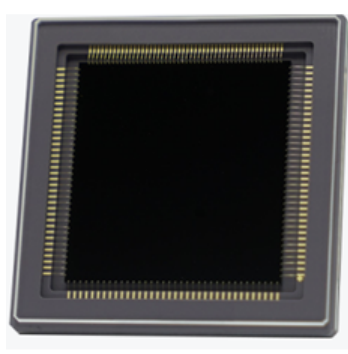

(a)

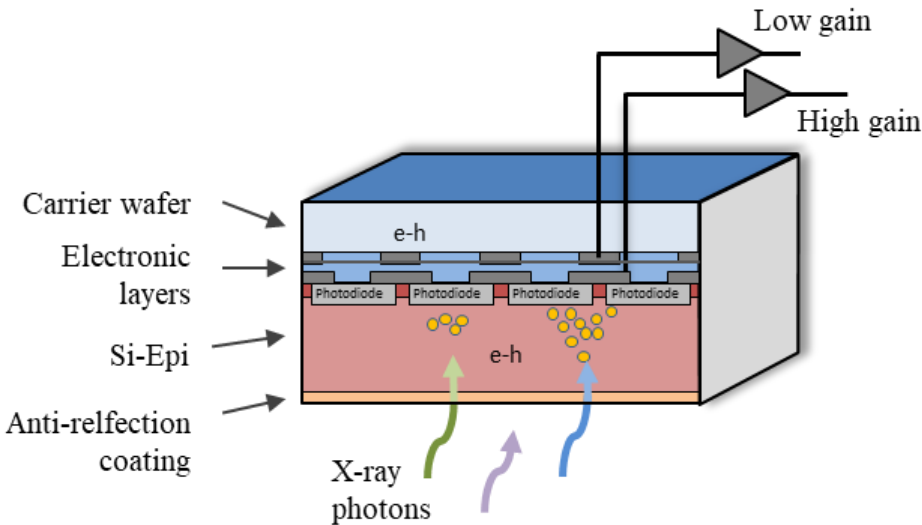

(b)

Figure 1: sCMOS GSENSE400BSI sensor (a) Sensor picture (Gpixel, http://gpixel.com) and (b) schematic cross section of the sensor

Table 1 Electro-optical characteristics of the GSENSE400BSI sensor specifications and TUCSEN Dhyana95 camera measurements results

\begin{tabular}{|c|c|c|c|}
\hline & Symbol & Value & ref \\
\hline Gain & $\mathrm{K}$ & Low gain, High gain or HDR mode & \multirow{6}{*}{$\begin{array}{l}\text { GPIXEL datasheet } \\
\text { (www.gpixel.com) }\end{array}$} \\
\hline Frame Rate & fps & $\begin{array}{l}24 \mathrm{~Hz} \text { Full frame (HDR) } \\
48 \mathrm{~Hz} \text { Full frame (STD) }\end{array}$ & \\
\hline $\begin{array}{c}\text { Readout } \\
\text { architecture }\end{array}$ & & Rolling Shutter & \\
\hline Pixel Size & $\mathrm{px}$ & $11 \times 11 \mu \mathrm{m}^{2}$ & \\
\hline Sensor size & & $\begin{array}{l}4 \mathrm{M}-2048 \times 2048 \text { pixels } \\
(22.5 \mathrm{~mm} \times 22.5 \mathrm{~mm})\end{array}$ & \\
\hline Exp. times & $\mathrm{t}$ & $20 \mu \mathrm{s}-10 \mathrm{~s}$ & \\
\hline Binning & & No & \\
\hline Readout noise & $\sigma_{\text {read }}$ & $<2 e-$ rms (HDR \& HG) \& < 45 e- rms (LG) & \multirow{5}{*}{$\begin{array}{l}\text { TUCSEN Dhyana95 } \\
\text { datasheet } \\
\text { (www.tucsens.com) } \\
\text { Wang et al., } 2017 \\
\text { Desjardins et al, } \\
2019\end{array}$} \\
\hline Dark noise & $\sigma_{\text {dark }}$ & $>3 \mathrm{e}-/ \mathrm{s} / \mathrm{pix}\left(-20^{\circ} \mathrm{C}\right)$ & \\
\hline $\begin{array}{l}\text { Full Well } \\
\text { Capacity }\end{array}$ & FWC & 30 ke- (HDR), 1700 e- (HG) \& > $80 \mathrm{ke}-(\mathrm{LG})$ & \\
\hline $\begin{array}{l}\text { Spatial pixel } \\
\text { offset noise }\end{array}$ & DSNU & $<5 \mathrm{e}-$ & \\
\hline $\begin{array}{l}\text { Spatial pixel } \\
\text { gain noise }\end{array}$ & PRNU & $<1 \%$ & \\
\hline
\end{tabular}

Following these specifications, the Signal to Noise Ratio (SNR) of the GSENSE400BSI, defined by equation 1 (Janesick, J. R., 2007, (with equation 3.11 revised as discussed in stackexchange web forum)], has been calculated the different modes and compared to the other similar sensors in the X-ray domain (Figure 2). 


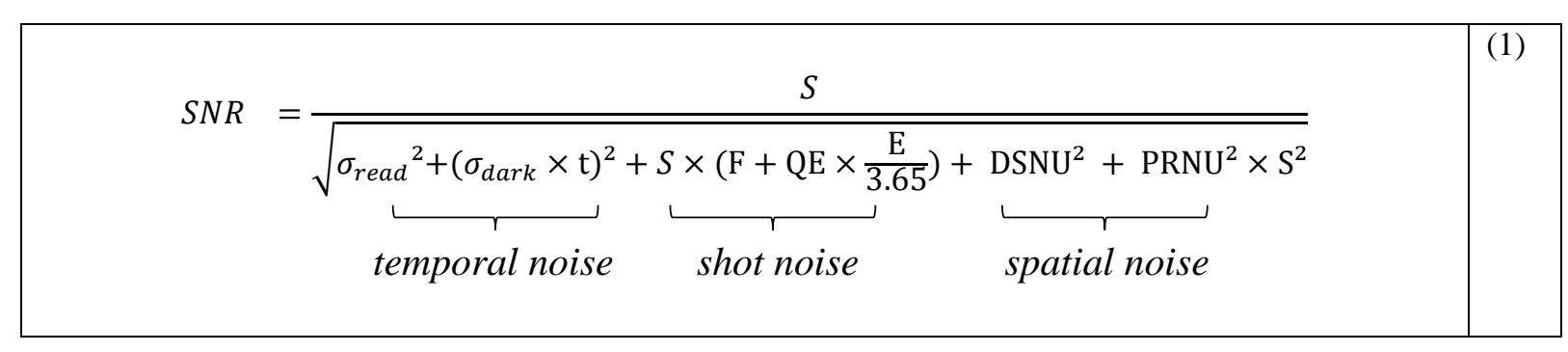

where $\mathrm{S}$ is the signal in (e-) equal to $Q E \times N \times \mathrm{E} / 3.65$ with QE is the Quantum Efficiency depending of the beam energy $\mathrm{E}, \mathrm{N}$ the number of photon, $3.65 \mathrm{eV}$ is the electron-hole pair creation energy in silicon, $\mathrm{F}$ the Fano factor ( 0.12 for silicon), $\sigma_{\text {readout }} \& \sigma_{\text {dark }}$ the temporal noises \& DSNU and PRNU the spatial noises as given in table 1 .

The figure 2 shows an example of the SNR for a X-ray beam of $500 \mathrm{eV}$ and its comparison with published results on others sensors, i.e. a back illuminated CCD (PIMTE2048B camera on https://www.princetoninstruments.com) and the PERCIVAL detector (Khromova et al, 2016). Here, the SNR estimations takes only into account the shot noise, temporal noise (thermal noise and readout noise) and the theoretical Quantum Efficiency (QE, see also §3.2) of each sensor. The spatial noise is excluded here (PRNU $=0$ and DSNU $=0)$. In this figure we also represent the photon detection limit (at $500 \mathrm{eV}$ ) corresponding to the full well capacity (in photon $/ \mathrm{mm}^{2}$ ) taking into account the pixel area (see table 2 for all details).

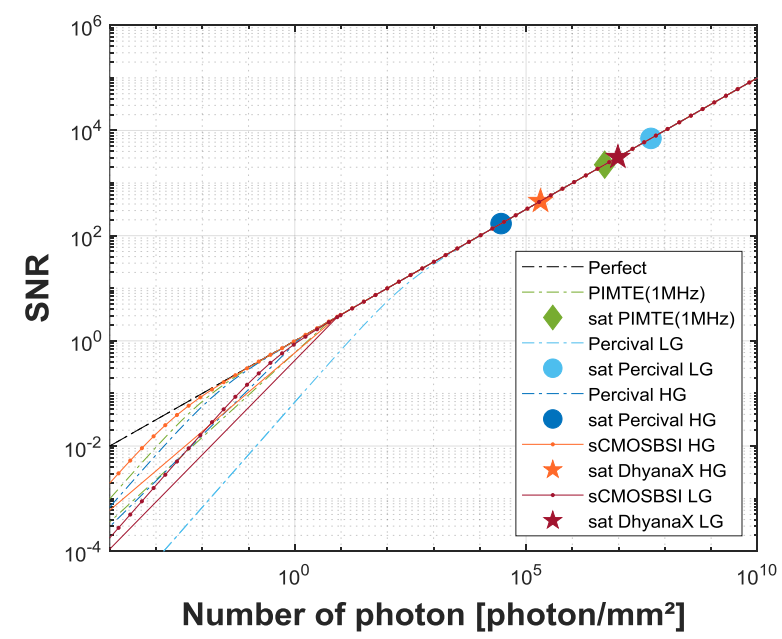

Figure 2 : Signal to Noise Ratio estimation for GSENSE400BSI (Low Gain and High Gain), PIMTE CCDBSI (with ADC speed of $1 \mathrm{MHz}$ ), PERCIVAL (Low Gain and High Gain) at $500 \mathrm{eV}$. The SNR of perfect detector is calculated for QE=1, noise $=0 \mathrm{e}-$ avec pixel size of $10 \mu \mathrm{m}$. The SNR of each detector are calculated from the details reported in table 2 and considering a dark noise given for $1 \mathrm{~s}$ of integration time. The saturation level of each compared sensors is given by dot, pentagon and star respectively for PERCIVAL, PIMTECCDBSI and GSENSE400BSI with a surface normalization.

\begin{tabular}{|l|l|l|l|}
\hline \multirow{2}{*}{ Detector } & $\begin{array}{l}\text { PI-MTE CCD BSI } \\
\text { (datasheet } \\
\text { PIMTE2048B) } \\
\text { [http://www.princeton.c } \\
\text { om] }\end{array}$ & $\begin{array}{l}\text { PERCIVAL (BSI) } \\
\text { Khromova et al, 2016) }\end{array}$ & $\begin{array}{l}\text { GSENSE400BSI } \\
\text { (Desjardins et al, 2019) }\end{array}$ \\
\hline Pixel size & $13.5 \times 13.5 \mu \mathrm{m}^{2}$ & $27 \times 27 \mu \mathrm{m}^{2}$ & $11 \times 11 \mu \mathrm{m}^{2}$ \\
\hline
\end{tabular}




\begin{tabular}{|c|c|c|c|}
\hline $\mathrm{QE}(500 \mathrm{eV})$ & 0.85 & $\sim 0.8$ (unpublished) & 0.5 up to 0.8 (see $\S 3.2)$ \\
\hline Readout noise & 10 e- rms (1MHz ADC) & $\begin{array}{l}1600 \text { e- rms (lowest gain) } \\
15 \text { e- rms (highest gain) }\end{array}$ & $\begin{array}{l}2 \text { e- rms (HG) } \\
38 \text { e- rms (LG) }\end{array}$ \\
\hline Dark noise & $0.02 \mathrm{e}-/ \mathrm{s} / \mathrm{pixel}\left(-50^{\circ} \mathrm{C}\right)$ & $\begin{array}{l}\text { Supposed low }\left(-40^{\circ} \mathrm{C}\right) \\
\text { (unpublished) }\end{array}$ & $3 \mathrm{e}-/ \mathrm{s} /$ pixel $\left(-20^{\circ} \mathrm{C}\right)$ \\
\hline FWC & $100 \mathrm{ke}-$ & $\begin{array}{l}\sim 4 \mathrm{Me}-/ \text { pixel (lowest gain) } \\
\sim 2000 \text { e- (highest gain) }\end{array}$ & $\begin{array}{l}80 \text { ke- (LG) } \\
30 \text { ke- (HDR) } \\
1600 \text { e- (HG) }\end{array}$ \\
\hline
\end{tabular}

Now considering the HDR mode (dark noise of $3 \mathrm{e}-/ \mathrm{s} / \mathrm{px}$, readout noise of $2 \mathrm{e}-\mathrm{rms}$ and $\mathrm{FWC}=30 \mathrm{ke}$-) and the theoretical QE (as describe in the next paragraph, see $\$ 3.2$ ), the limit of detection (SNR = 1) and the pixel saturation of the GSENSE400BSI sensor can be evaluated and the dynamic range (DR=FWC/limit) can be estimated in function of the beam energy (figure 3)

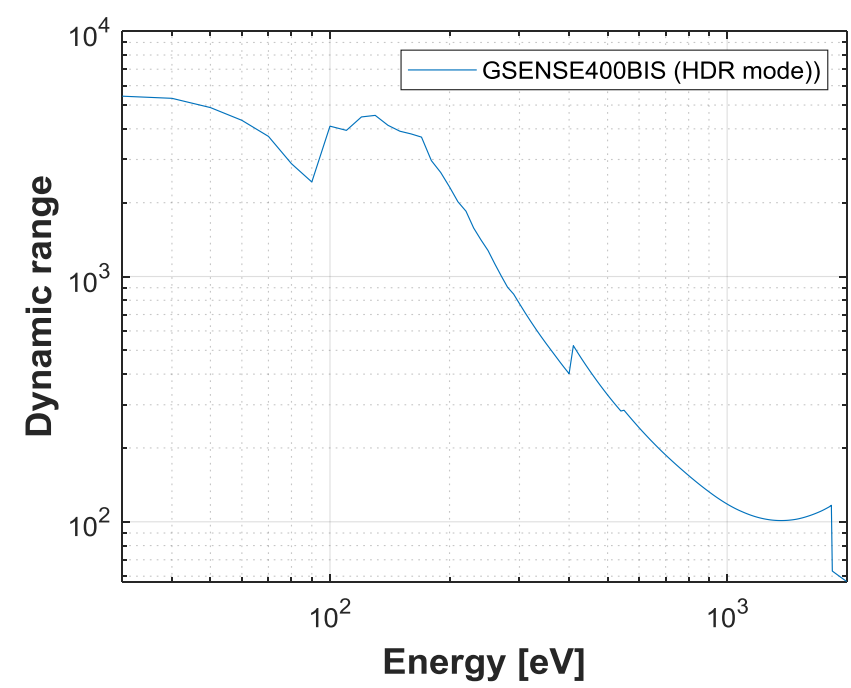

Figure 3 : Theoretical dynamic range of the GSENSE400BSI (HDR mode) considering the X-ray photon limits of detection (SNR=1) and the X-ray saturation limit (diamond red line).

\subsection{X-ray camera design and software integration}

In order to easily evaluate and integrate the sCMOS GENSE400BSI sensor on the soft Xray beamlines of SOLEIL synchrotron, we have adapted a TUCSEN® Dhyana95 camera, 
which has been totally disassembled and reassembled into a home-made mechanics (

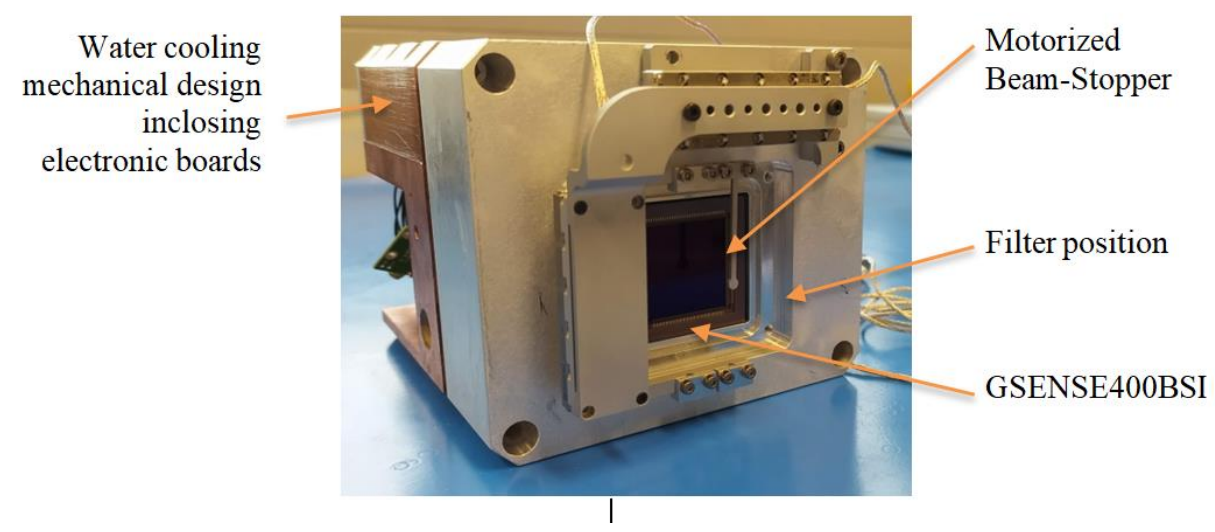

Figure 4), to make it compatible as much as possible in vacuum environment ( $\left.10^{-6} \mathrm{mbar}\right)$. It can be noted that the components vacuum compatibility (cables, cooling, PCB board, ...) have not been the subject of in-depth studies for this first prototype assembly, whereas it is a crucial technical point which must be taken into account for the future detector that will be permanently installed on SOLEIL beamlines. Indeed, as seen during our series of tests, the cooled sensor could be quickly contaminated which could degrade its efficiency (Deiries et al, 2016). Then, following components are located in vacuum: sensor, sensor board, DAQ board (FPGA, power supply, data transfer, ...) and a water-cooling circuit $\left(>12^{\circ} \mathrm{C}\right)$ has been made to maintain an optimal temperature of both the sensor $\left(-20^{\circ} \mathrm{C}\right.$ with 2 stages of Peltier cooler) and the electronic components. As typically done for such soft X-ray experiment, the assembly has been designed to have a perfect tightness in parasite light with the possibility to add different visible filter (for example, carbon or alumina filter from Luxel®, https://luxel.com $)$ in front of the sensor. It remains compact $(10 \mathrm{~cm} \times 11 \mathrm{~cm} \times 12 \mathrm{~cm})$ to fit with a vacuum chamber on beamline and their restrictive environment. Furthermore, an X-ray beam stop could be added in front of the camera and positioned with two linear piezo motorizations as shown in Figure 4 (Popescu et al 2019).

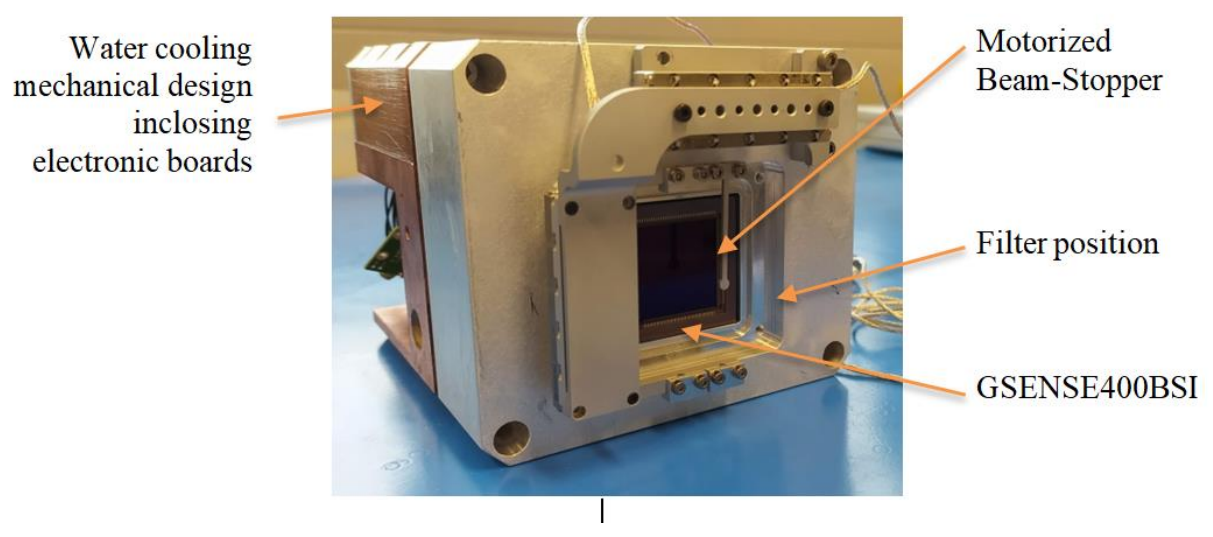


To transfer and to record the images, this camera is connected via a USB3 interface through a dedicated UHV USB3 feedthrough to a classical Windows 64bit computer equipped with a Tango LIMA device (https://www.tango-controls.org) based on TUCAM API from TUCSEN®. Then, this camera keeps the same characteristics as the initial camera in term of SNR, readout speed $(24 \mathrm{~Hz}$ full frame), cooling and synchronization capacities.

\section{Characterization tests results and discussion}

\subsection{X-ray experimental setup}

As it has been already presented in the first DhyanaX prototype setup (Desjardins et al, 2019), this X-ray camera has been mounted in the reflectometer of the XUV branch of the METROLOGIE beamline at SOLEIL synchrotron. The beamline allows to select a photon beam in the soft X-ray range $(30 \mathrm{eV}-2000 \mathrm{eV})$ using several combination of gratings and mirrors to optimize the beamline transmission and energy resolution and a series of thin filters llowing to reduce the harmonics contamination in each configuration. The beam size could also be easily adjusted and a calibrated photodiodes (IRD® AXUV100, https://optodiode.com) can be used measuring, with less than $10 \%$ of uncertainty, the incident $\mathrm{X}$-ray beam flux at each energy. With this configuration, the QE of two versions of GSENSE400BSI has been measured (\$3.2) over the full soft X-ray range. In addition, to characterize fully the detector, we have measured the spatial resolution at $1500 \mathrm{eV}(\$ 3.3)$, the energy resolution (§3.3) as well the sensor radiation hardness (§3.4).

\subsection{GSENSE400BSI soft X-ray Efficiency}

Two versions of sCMOS GSENSE400BSI versions have been evaluated: the GSENSE400BSI-TVISB, the standard visible light dedicated sensor version and the GSENSE400BSI-GP, an experimental version dedicated for UV range (Harada et al., 2019) recently developed before a new mass production. The backside, as represented on the sensor 


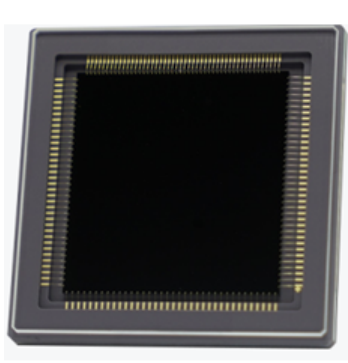

(a)

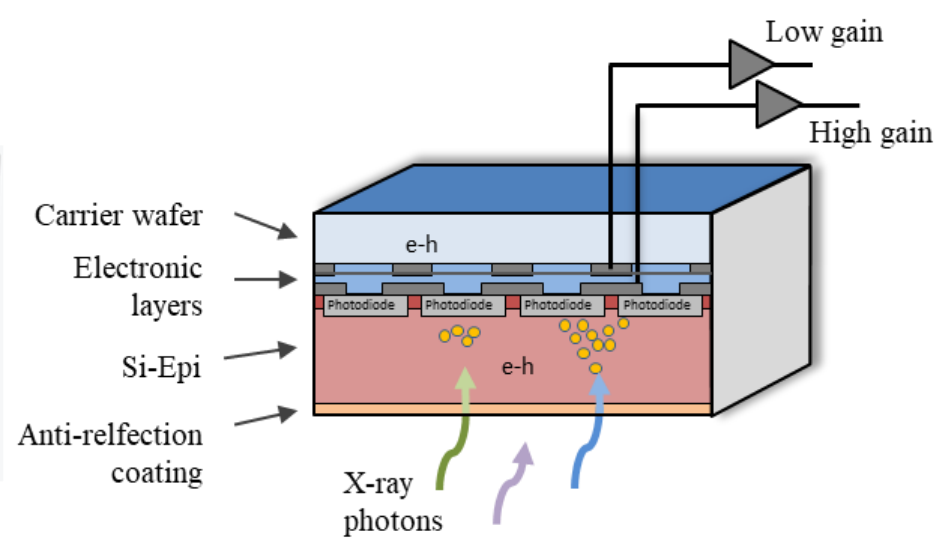

(b)

Figure 1 (b), is composed by a silicon epitaxial layer (Si-Epi) of $10 \mu \mathrm{m}$ thick, $7 \mathrm{~nm}$ of SiO2 followed by $63 \mathrm{~nm} \mathrm{Si3N4}$. This silicon nitride is on the top of the sensor, both layers serve as an anti-reflection coating (ARc) purpose and passivation layer (originally processed for visible applications). The second sensor tested has no anti-reflective coating (no-ARc) on the photo-sensitive area but has a thinner Si-Epi layer thickness around $4 \mu \mathrm{m}$. The method and measurement of the QE of the sensor have already been reported in the previous work (Desjardins 2019). The final result for both sensors is presented in figure 5 where the measurements have been superimposed with model results proposed in equation 2;

$$
\begin{aligned}
Q E_{\text {model }}(E)= & e^{-z_{S i O} \times \mu_{S i O}(E)} \times e^{-z_{S i N} \times \mu_{S i O}(E)} \times e^{-z_{\text {neutral }} \times \mu_{S i}(E)} \\
& \times\left(1-e^{-e^{-z_{\text {depleted }} \times \mu_{S i O}(E)}}\right)
\end{aligned}
$$

where the $\mathrm{z}_{\mathrm{SiO}}$ and $\mathrm{z}_{\mathrm{SiN}}$ are the thickness material of the AR coating, $z_{\text {neutral }}$ and $\mathrm{z}_{\text {depleted }}$ are the thickness of the non-sensitive and sensitive silicon layer respectively. $\mu \mathrm{SiO}, \mu \mathrm{SiN}$ and $\mu \mathrm{Si}$ are the attenuation coefficient for these different elements. 


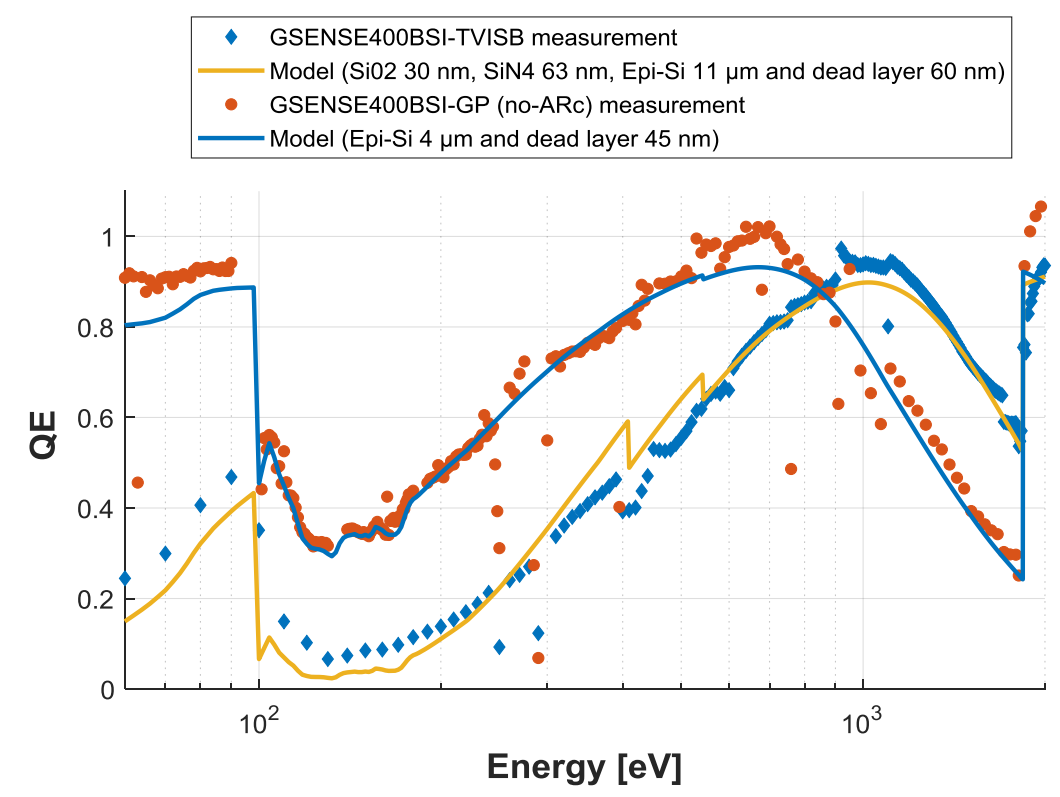

Figure 5 : GSENSE400BSI-TVISB and GSENSE400BSI-GP Quantum Efficiency measurements compared to a theoretical model given by AR coating and Si-Epi absorption.

To fit with the QE measurement results, the different parameters of the model (Equation 2) have been adjusted. For the GSENSE400BSI-TVISB, the Silicon oxide thickness has been increased to $30 \mathrm{~nm}$ certainly due to the contamination and oxidation at its surface, the dead layer has been adjusted to $60 \mathrm{~nm}$ and the Si-Epi to $11 \mu \mathrm{m}$. Whereas the GSNESE400BSI-GP model fit correctly with the sensor parameters with a dead layer of $45 \mathrm{~nm}$ and a Si-Epi around $4 \mu \mathrm{m}$ (and no-AR coating layer). Both measurements and models are reported in the figure 5 .

Our result shows that the QE of the classical GSENSE400BSI is highly sufficient for a wide range of high flux synchrotron applications. In order to illustrate its low energy efficiency, a diffracted pattern image form a $5 \mu \mathrm{m}$ diameter pinhole has been performed at an energy beam of $186 \mathrm{eV}$ with the sCMOS GSENSE400BSI-TVSIB and the result is showed in figure 6.

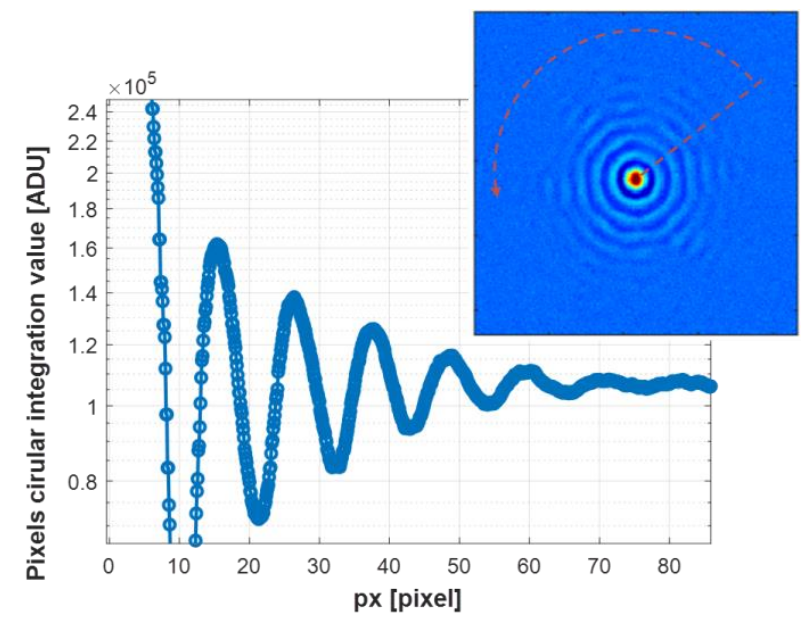


Figure 6 : Circular integration of pinhole diffraction image from SCMOS GSENSEBSI400 obtained at $186 \mathrm{eV}$ on METROLOGIE Beamline (100 accumulations of 100 ms dark-corrected images in HDR mode).

With twice the efficiency around $300 \mathrm{eV}$, the no-ARc sensor (GP version) could be a better choice for the so-called water window's region that include $\mathrm{C}, \mathrm{N}$ and $\mathrm{O} \mathrm{K}$ edge, but it should be noted that it will be at the price of using the thin Si-Epi layer sensor more sensible to radiation damage, as it will be shown in paragraph 3.4.

\subsection{GSENSE400BSI spatial \& energy resolution}

The sCMOS GSENSEBSI400-TVISB spatial resolution has been checked using the ISO knife slanted edge method (International Organization for Standardization [ISO], 2000). A silicon nitride support films has been used as edge slightly tilted (the edge angle was about $10^{\circ}$ off of the pixel array alignment) and illuminated with X-ray beam at $1500 \mathrm{eV}$. The Modulation Transfer Function (MTF) has been calculated from cropped dark-corrected image (figure 7) carried out by using sfrmat3 Matlab script (Burns, 2009). The result is given in pair of line per $\mathrm{mm}$ in figure 7.

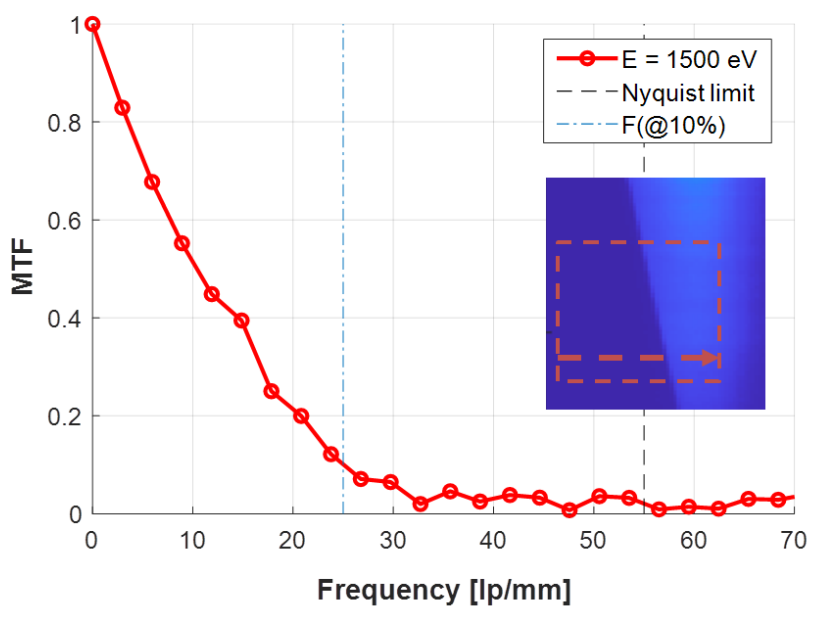

Figure 7 : MTF calculation from sCMOS GSENSEBSI400 slanted edge image at $1500 \mathrm{eV}$ on METROLOGIE Beamline (1000 accumulation of dark corrected images of $100 \mathrm{~ms}$ ).

A $10 \%$ modulation transfer contrast is reached around $24 \mathrm{lp} / \mathrm{mm}$, corresponding to a halfpitch resolution of $21 \mu \mathrm{m}$, in good agreement with the sensor pixel size $\left(11 \mu \mathrm{m}^{2}\right)$. This resolution could be degraded for lower X-ray energy due to the lateral charge diffusion within the thick Epi-Silicon $(\sim 10 \mu \mathrm{m})$ and the neutral layer, but it was not the main object of our in 
this work. However, this effect will be discussed (figure 8b) in the next paragraph where we described the sensor energy resolution.

In order to characterize the energy resolution of the detector, a GSENSE400BSI-GP sensor has been exposed by a small part (out of focus) monochromatic X-ray beam of METROLOGIE beamline with very low flux (no more than $10^{6} \mathrm{ph} / \mathrm{s}$ ) in order to collect images of single isolated events. For each energy, a large series of cropped images $(100 \times$ 2048 pixels) has been acquired with the HDR mode and for a short exposure (20 $\mu$ s, 300 fps). This type of measurement, widely used in X-ray Spectroscopy Imaging and X-ray Spectroscopy astronomy (Lung et al., 1984) allows to work similarly as a counting detector with few photons per pixel per second. Then, for every dark-corrected image recorded, the single and isolated photon events (highlighted by blue circles in figure $8 \mathrm{a}$ ) have been extracted and their amplitude cumulated to obtain a spectrum. This algorithm was carried out using in a MATLAB script isolated a pixel if its value is $95 \%$ greater than the sum of the 8 neighboring pixels. The result is displayed in figure $8 \mathrm{~b}$ in which we superposed the normalized spectrums for energy from $100 \mathrm{eV}$ to $1500 \mathrm{eV}$. In order to convert the spectra in $\mathrm{eV}$ the overall gain of $0.67 \mathrm{ADU} / \mathrm{eV}$, calculated from this measurement, has been applied.

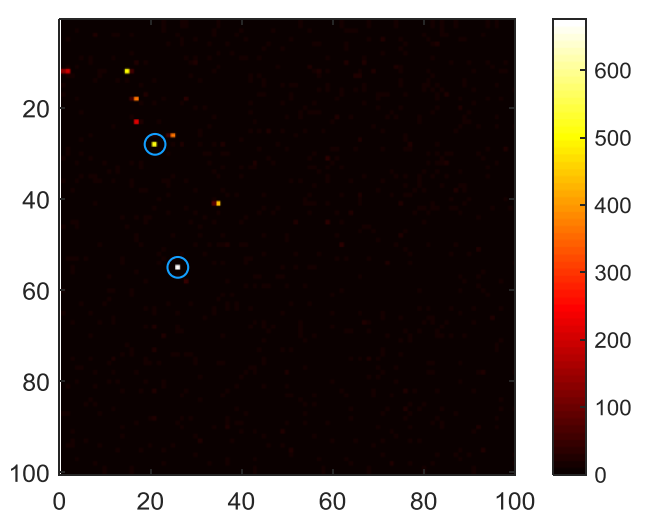

(a)

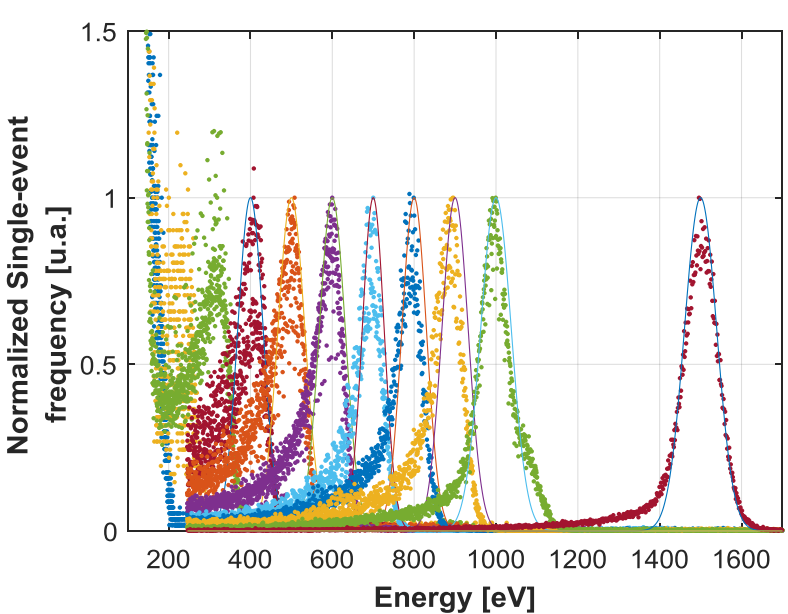

(b)

Figure 8 : (a) Example of image analyzed for X-ray energy beam of $1000 \mathrm{eV}$ (blue circles are the pixel extracted). (b) Spectrums of single-event frequencies for X-ray photon energy of 100-1500 eV. The spectrums for $400 \mathrm{eV}$ to $1500 \mathrm{eV}$ have been voluntary cropped in low-energy range (down to $200 \mathrm{eV}$ ).

The energy resolution is estimated by the full width at half maximum (FWHM) of the energy peaks (Janesick, 2001) and given in the figure 9 where the theoretical limitation given by the equation 3 below is also added for comparison: 


$$
F W H M(e V)=2.35 \times 3.65 \times \sqrt{F \times E / 3.65+\sigma_{\text {SCMOS }}^{2}}
$$

where $\mathrm{F}$ is the Fano factor ( 0.1 for silicon), $\mathrm{E}$ the photon energy, $3.65 \mathrm{eV}$ is the electronhole pair creation energy for X-ray absorption in silicon and $\sigma_{S C M O S}^{2}$ is the quadratic sum of noises of the sCMOS camera.

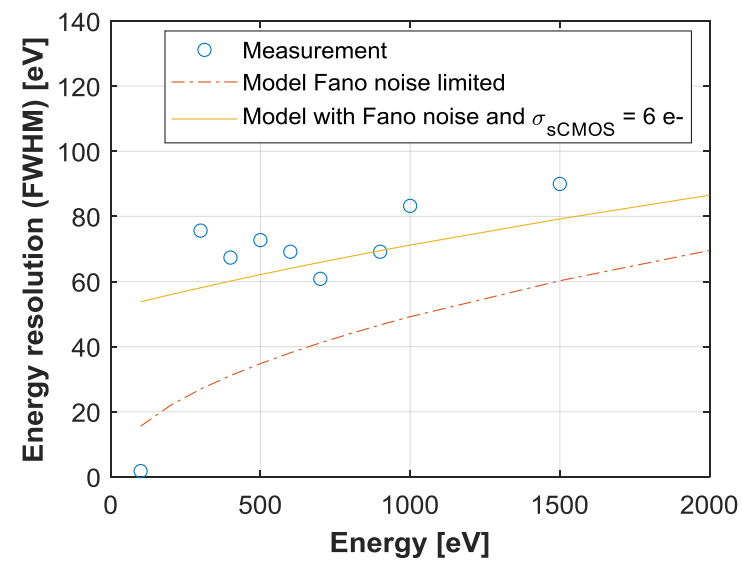

Figure 9 : X-ray energy resolution of GSENSE400BSI-GP measurement results compared to the Fano noise limit and to the fit FHWM with $\sigma_{\mathrm{scMOs}}=6 \mathrm{e}-$.

We find an energy resolution that is slightly higher of $20 \mathrm{eV}$ than the theoretical one, due to an excess noise in this camera prototype (with readout noise $>2 \mathrm{e}-\mathrm{rms}$ and higher offset and gain fixed-pattern noise). Furthermore, the sensor cooling was set to $16^{\circ} \mathrm{C}$ (only water cooling was operated and the Peltier stage was deactivated). In figure 9 is superposed a FWHM model fitting the datas with $\sigma_{S C M O S}^{2}$ of 6 e- rms. Also, the noise floor, represented in figure 8 by the tail on the energy peaks is reached for energy below $200 \mathrm{eV}$. As stated above, the effect is attributed to the spread charge created close to the surface into the thick Si and particularly in the non-depleted region.

\subsection{GSENSE400BSI Radiation hardness}

Considering the typical requirement for an X-ray detector needed at SOLEIL with energy of photon between 40 to $2000 \mathrm{eV}$ and a monochromatic flux largely exceeded $10^{12} \mathrm{ph} / \mathrm{s} / 0.01 \% \mathrm{BW}$, radiation hardness has been evaluated on both sensors on the METROLOGIE beamline for different energies and X-ray fluences. 


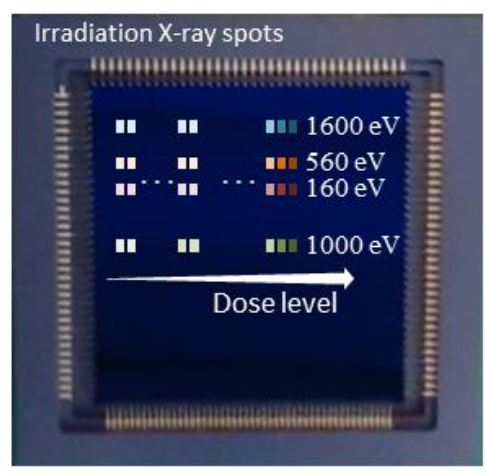

(a)

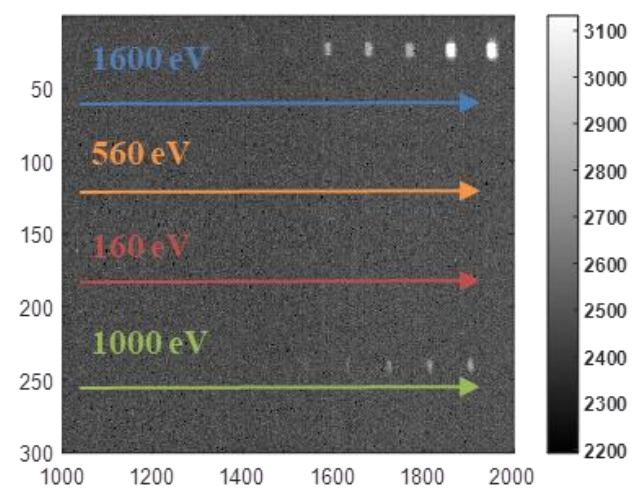

(b)

Figure 10 : (a) Schematic representation of X-ray irradiation of GSENSEBSI400-TSVIB for $160 \mathrm{eV}, 560 \mathrm{eV} 1000 \mathrm{eV}$ and $1600 \mathrm{eV}$ (b) Part of GSENSEBSI400-TSVIB dark image (300 px by $1000 \mathrm{px}$ ) after irradiation (contrast voluntary increased)

The GSENSEBSI400-TVISB sensor has been locally exposed to a direct, collimated $(<200$ $\times 200 \mu^{2}$ ) and monochromatic X-ray beam of $160 \mathrm{eV}, 560 \mathrm{eV}, 1000 \mathrm{eV}$ and $1600 \mathrm{eV}$ and the GSENSE400BSI-GP only by a lower energy X-ray beam of $80 \mathrm{eV}$. For each energy, the delivered $\mathrm{X}$ dose has been increased by increasing the time of irradiation from few seconds to several hours (figure 10.a). The absorbed dose (D), i.e. the amount of energy absorbed per unit of mass of a given material (Ravotti, 2018), is basically calculated by using the following equation :

$$
D(z)=\frac{\mu}{\rho} \times \Phi / A \times t \times E
$$

with $\Phi$ is the considered X-ray photon flux, $A$ is the surface exposed by the monochromatic X-ray beam with energy $E, \mu / \rho$ the mass energy absorption coefficient (of $\mathrm{Si}$ ) and $\mathrm{t}$ the exposure time.

The table 3 gives the absorbed dose evaluated on both the surface and the electronic layers of the sensor for different incident and different energies.

\section{Table 3. Soft X-ray irradiation details}

\begin{tabular}{|l|l|l|l|}
\hline Energy & $\begin{array}{l}\text { Incident flux }\left(\Phi_{0}\right) \text { on the sensor } \\
\text { (Total exposure time) }\end{array}$ & TID on surface & TID on electronic layer $^{2}$ \\
\hline
\end{tabular}

1 The TID (absorbed dose) on the surface is estimated from equation 4 considering the anti-reflection layers thickness attenuation and with $\mu / \rho$ extracted from NIST FFAST database (https://physics.nist.gov) 2 The TID (absorbed dose) on the electronic layer is estimated from equation 4 considering the antireflective layer and Epi-Si thicknesses attenuation and with $\mu / \rho$ extracted from NIST FFAST database (https://physics.nist.gov) 


\begin{tabular}{|l|l|c|l|}
\hline GSENSE400BSI-TVISB \\
\hline $160 \mathrm{eV}$ & $4.1 \times 10^{8} \mathrm{ph} / \mathrm{s}(2 \mathrm{~s}$ to $1 \mathrm{~h})$ & $25 \mathrm{kGy}$ to $8 \mathrm{MGy}$ & Negligible $\left.\left(\mathrm{T}_{\text {Si-Epi }(10 \mu \mathrm{m}}\right)=0\right)$ \\
\hline $560 \mathrm{eV}$ & $2.0 \times 10^{7} \mathrm{ph} / \mathrm{s}(7 \mathrm{~s}$ to $0.5 \mathrm{~h})$ & $64 \mathrm{~Gy}$ to $114 \mathrm{kGy}$ & Negligible $\left(\mathrm{T}_{\text {Si-Epi }}=0\right)$ \\
\hline $1000 \mathrm{eV}$ & $1.7 \times 10^{6} \mathrm{ph} / \mathrm{s}(1 \mathrm{~s}$ to $3 \mathrm{~h})$ & $4 \mathrm{~Gy}$ to $44 \mathrm{kGy}$ & $0.1 \mathrm{~Gy}$ to $1 \mathrm{kGy}$ \\
\hline $1600 \mathrm{eV}$ & $1.2 \times 10^{7} \mathrm{ph} / \mathrm{s}(4 \mathrm{~s}$ to $5 \mathrm{~h})$ & $45 \mathrm{~Gy}$ to $180 \mathrm{kGy}$ & $16 \mathrm{~Gy}$ to $64 \mathrm{kGy}$ \\
\hline GSENSE400BSI-GP & $15 \mathrm{kGy}$ to $13 \mathrm{MGy}$ & $23 \mathrm{~Gy}$ to $20 \mathrm{kGy}$ \\
\hline $80 \mathrm{eV}$ & $3.010^{8} \mathrm{ph} / \mathrm{s}(0 \mathrm{~s}$ to $24 \mathrm{~h})$ &
\end{tabular}

We select characteristic energies over the wide soft x-ray energy range that allows to evaluate the radiation damage at the different layers on the sensor's thickness. Indeed, as shown in figure 11, the low x-ray photons are largely absorbed on the thin thickness of Epi-Si when the $\mathrm{X}$-ray photons above $1000 \mathrm{eV}$ goes through the Epi-Si and reach the electronic layer (figure 1b). Also, the photon beam at $80 \mathrm{eV}$ could penetrate the thinner Epi-Si of the GSENSE400BSI-GP $(4 \mu \mathrm{m})$ with a transmission about $0.1 \%$, against below $10^{-5} \%$ for the GSENSE400SI-TVISB $(10 \mu \mathrm{m})$.

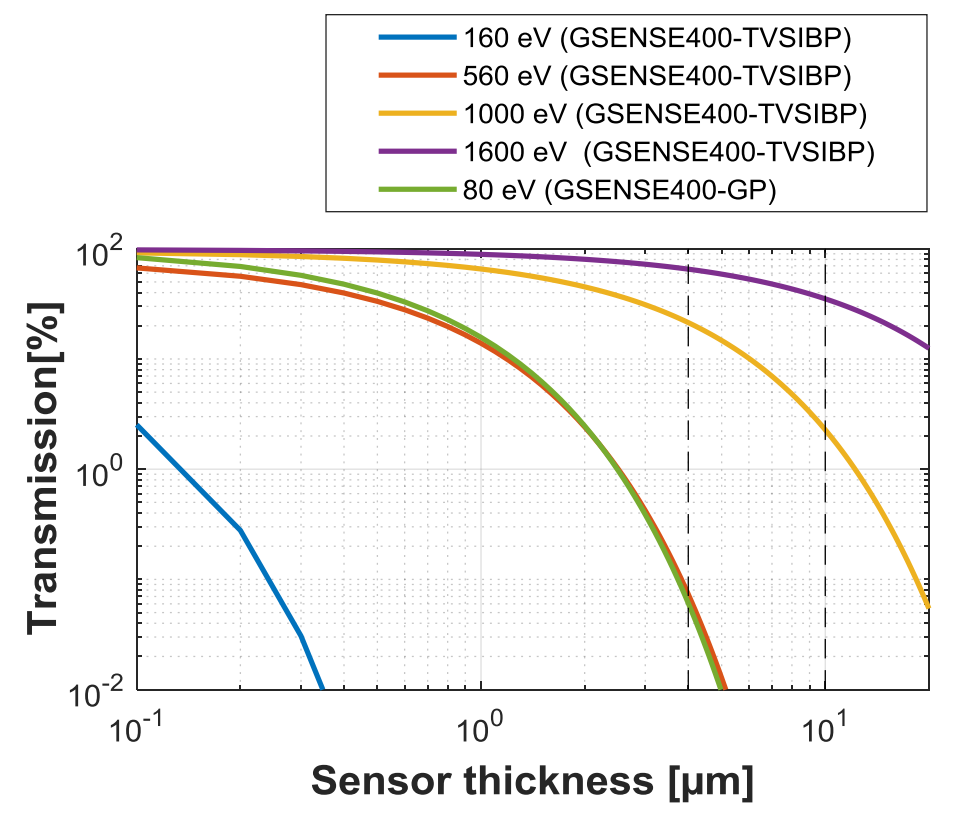

Figure 11 : Theoretical transmission versus the Si thickness for X-ray photon of $160 \mathrm{eV}, 560 \mathrm{eV}, 1000 \mathrm{eV}$ and $1600 \mathrm{eV}$ through the GSENSEBSI400-TVISB and of $80 \mathrm{eV}$ through the GSENSEBSI400-GP.

As it can be found elsewhere (Clarke, 1994, Hancock et al., 1997), one of the radiation effect on the sensor manifests as an increased leakage current (pixel offset level), consequently we have compared the dark image after irradiations to the original one for the same exposure time, as showed in figure 10b. The mechanisms of these effects are complex and are not studied here but the results extracted from the figure 10.b are discussed. As described above, the low X-ray energies irradiation series $(160 \mathrm{eV}$ and $560 \mathrm{eV})$ with an absorbed dose up to 1 MGy into the surface layers of the GSENSE400TVSIB has no impact on the pixels offset 
level while the high energies X-ray irradiation series (1000 eV and $1600 \mathrm{eV})$ showed a significant increase of electronic offset level (for a lower dose) on the area irradiated (white spots on the right side of the dark image in figure 10.b). For these energies, the photons not absorbed by Epi-Si could penetrate deeply in the oxide and electronic layers of the CMOS sensor giving rise to a relatively important and persistent damage on the pixel (offset dark level remains high after few weeks at room temperature). It was similarly on the GSNES400BSI-GP with the $80 \mathrm{eV}$ beam impact.

Then, the pixels dark level evolution ( $\left.I_{\text {dark after irration. }}-I_{\text {dark } 0}\right)$ has been compared to the dose on electronic layer for all irradiation series to extract an estimation of the limit of dose acceptable (figure12 by the sensor.

\begin{tabular}{|c|c|}
\hline & \\
\hline (a) & (b) \\
\hline
\end{tabular}

Figure 12 : Radiation tolerance evaluation of (a) sCMOS GSENSE400BSI-TVSIB and (b) SCMOS GENSE400BSI-GP by excess dark measurement after soft X-ray irradiation with beam of $560 \mathrm{eV}, 1000 \mathrm{eV}$ and $1600 \mathrm{eV}$ and $80 \mathrm{eV}$, respectively. In dashed and red curves are the empirical model (equation 5 ).

The dark level increase, due to the dose, could be fitted with an empirical formula as:

$$
I_{\text {dark }}-I_{\text {dark } 0}=\left\{\begin{array}{r}
0, D<D_{t h} \\
K \times \log _{10}\left(\frac{D}{D_{t h}}\right), D \geq D_{t h}
\end{array}\right.
$$

where $\mathrm{D}$ is the dose on the electronic layer (equation 4 ), $\mathrm{D}_{\text {th }}$ is the dose threshold and $\mathrm{K} \mathrm{a}$ proportionality factor given the offset pixel evolution per (ADU/decade).

The fitted curve, in figure 12.a, for the two high energies measurement showed a limitation around $\mathrm{D}_{\text {th }}=200 \mathrm{~Gy}(20 \mathrm{kRad})$ and $\mathrm{K}=125 \mathrm{ADU} / \mathrm{dec}$. This dose threshold seems to be confirmed on the series of measurement performed on the thinner GSENSE400BSI-GP but with a higher dark evolution proportionality factor around $4000 \mathrm{ADU} / \mathrm{dec}$, in figure 12.b.

If the dose limit seems not very high, it's very difficult to compare with other similar devices as this measurement is not easy to do or easy to exploit and so rarely published, nevertheless, $\mathrm{e} 2 \mathrm{v}$ reports a radiation tolerance of $10 \mathrm{kRad}(100 \mathrm{~Gy})$ on the classical CCD design [datasheet du CCD40-42, D. Burt et al, 2009]. From this value $\left(D_{t h}=200 \mathrm{~Gy}\right)$, we represent in figure 13 an estimation of the limit of fluence (number of photons/unit area/time) to avoid non reversible damage of the two sensors tested here. 
Figure 13 : Limit of fluence $\left[\mathrm{ph} / \mathrm{s} / \mathrm{mm}^{2}\right]$ calculated for a limit the dose of $200 \mathrm{~Gy}$ on electronic layer of a GSENSE400BSITVSIB before damaging.

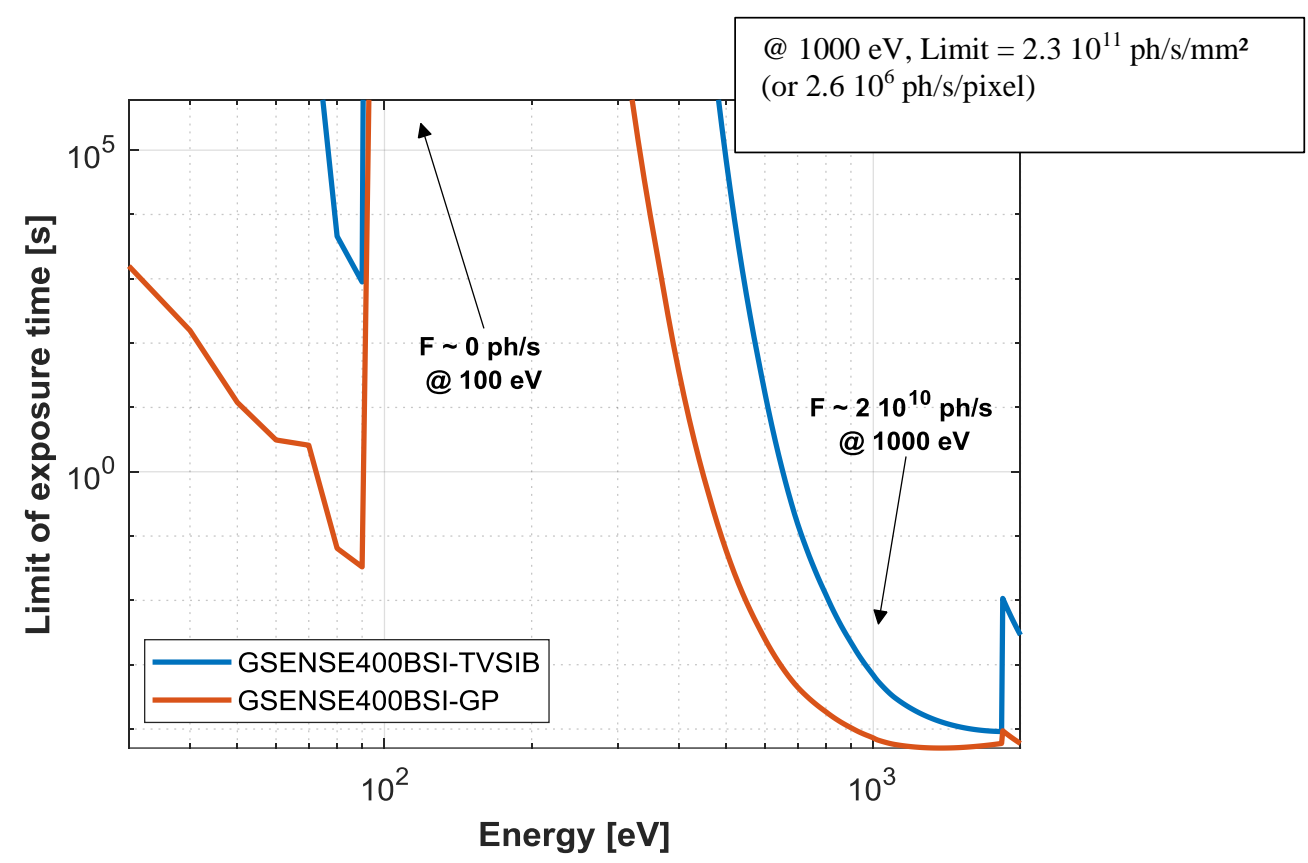

Figure 14 : Exposure time limit calculated for a limit the dose of $200 \mathrm{~Gy}$ on electronic layer of a GSENSE400BSI-TVSIB (blue) and GSENSE400BSI-GP (red) before damaging.

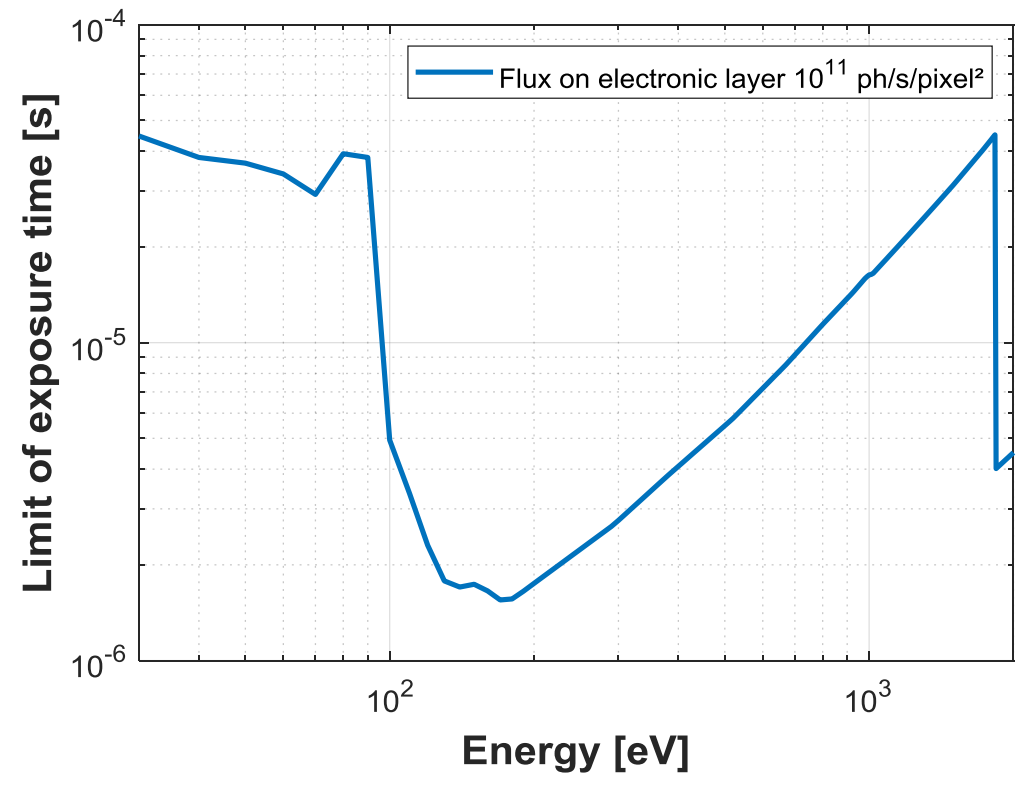

Figure 15 : Exposure time limit calculated for a limit the dose of $200 \mathrm{~Gy}$ with a flux of $10^{\mathrm{s}} \mathrm{ph} / \mathrm{s} /\left(11 \times 11 \mu \mathrm{m}^{2}\right)$ on electronic layer of a GSENSE400BSI.

4. Experiments performed on Soft X-ray beamlines using the SCMOS GSENSE400BSI 


\subsection{Soft X-ray Resonant Magnetic Scattering}

The GSENSE400BSI camera has been firstly installed on the RESOXS (Jaouen et al, 2004) end-station of the SEXTANTS beamline at SOLEIL. In order to illustrate the capabilities of the detector, a soft X-ray (400 eV to $2000 \mathrm{eV}$ ) Resonant Magnetic Scattering (XRMS) (Chauleau et al, 2018) experiment on magnetic samples exposed to the soft X-ray beam was performed.

As mentioned above in this paper, high resolution, low noise and high charge collection capacity are strongly required for this type of acquisition and are achieved up to now with a direct CCD detection. The beamline routinely uses with users the Back-Illuminated CCD PIMTE from Princeton Instrumentation (4Mpixels e2V CCD 42-40, pixel size $13.5 \mu \mathrm{m}$, QE $>90 \% @ 700 \mathrm{eV}, \mathrm{FWC}=100 \mathrm{ke}-$, electronic noise of $10 \mathrm{e}-\mathrm{rms}$ with $0.22 \mathrm{~Hz}$ ). In order to experimentally compare the performances of the sCMOS camera with this CCD camera, soft $\mathrm{X}$-ray scattering pattern from known a lithography prepared assembly of $\mathrm{X} \mu \mathrm{m} * \mathrm{X} \mu \mathrm{m}$ square $\mathrm{Co} / \mathrm{Cu}$ multilayer sample have been acquired.

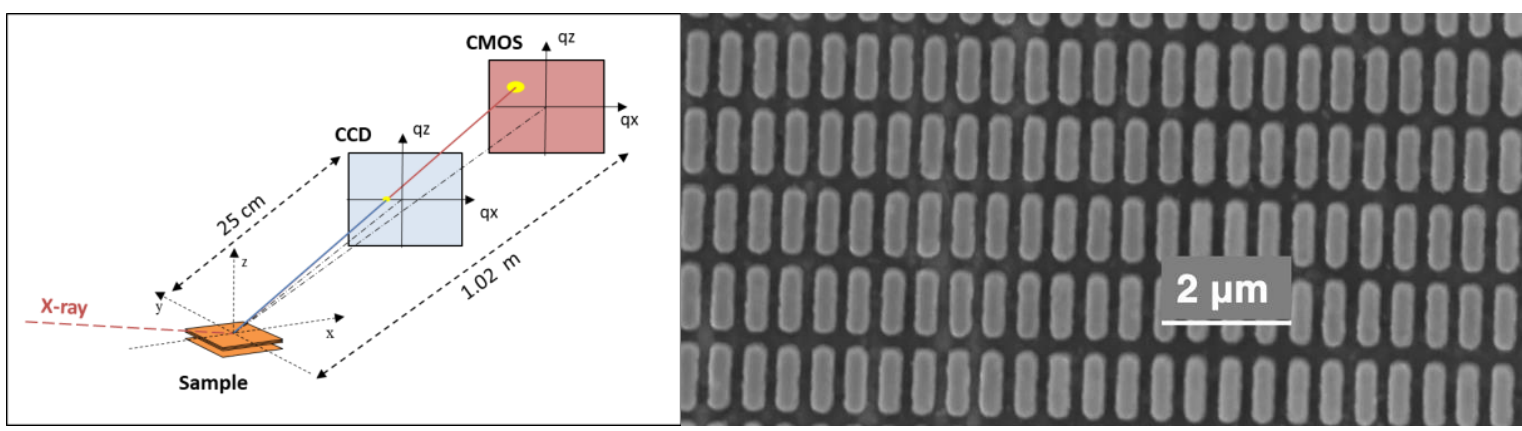

Figure 16 : SEXTANTS beamline synoptic for GSENSE400BSI camera test versus CCD installed. SEM image of the Co/Cu nanostrured sample of $530 \times 1220 \mathrm{~nm}$ rectange.

Unfortunately, the GSENSE400BSI camera has not been installed at the exactly same position compared to the CCD (figure 15). While the sCMOS was placed $1 \mathrm{~m}$ downstream from the sample, the CCD was placed closer, at distance of $25 \mathrm{~cm}$, which, considering the sensor area, limits the angular aperture from $1.5^{\circ}$ (sCMOS) to $6.3^{\circ}$ (CCD). Despite these nooptimized test conditions, two similar acquisitions have been performed on the both sensor and following these characteristics: exposure time of $200 \mathrm{~ms}$, accumulation of 100 images, HDR mode for GSENSE400BSI and ADC $1 \mathrm{MHz}$ mode for the CCD. The resulting images represented in angle $(\theta)$ are given in figure 16 for the same scale. 

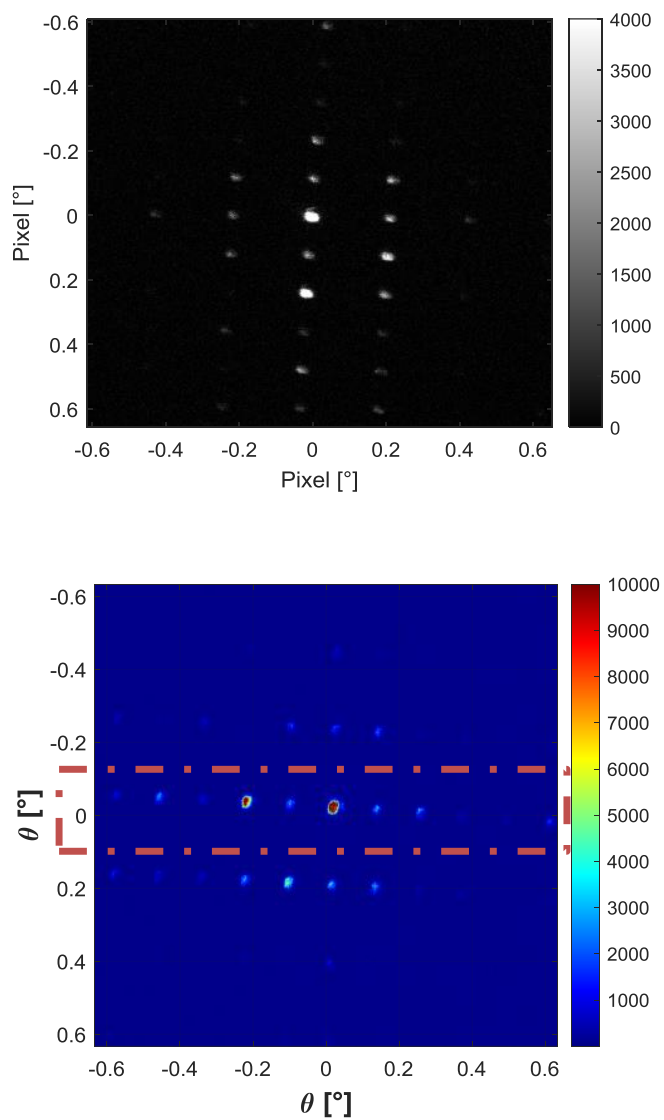

(a)
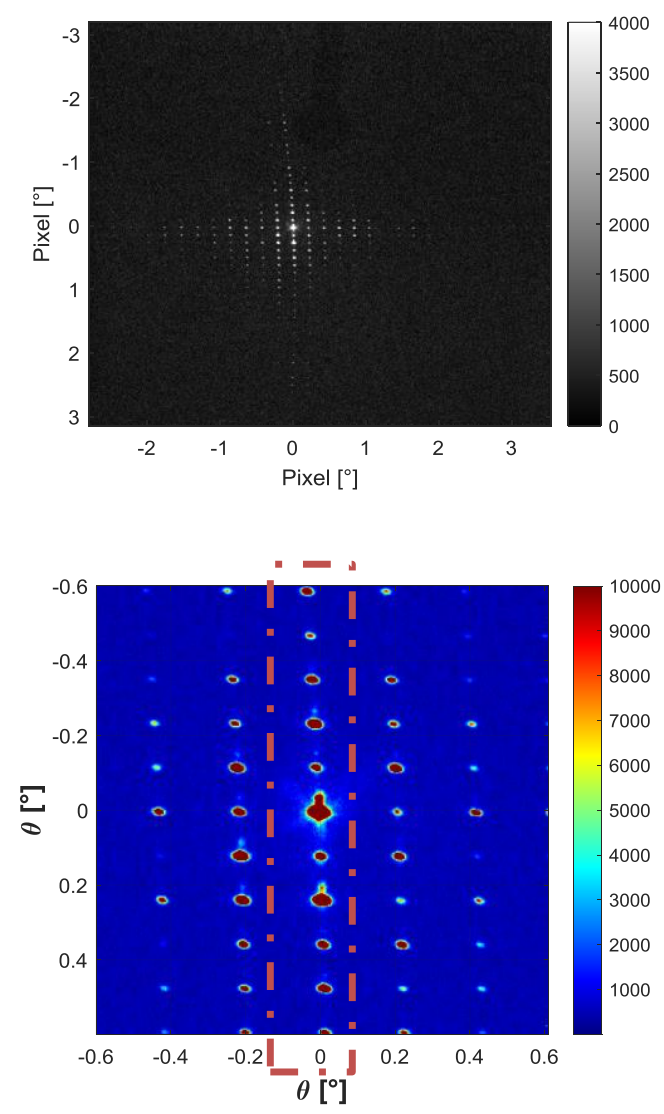

(b)

Figure 17 : Dark corrected images of soft X-ray scattering from Co/Cu multilayer sample at $767 \mathrm{eV}$ acquired with (a) the sCMOS GSENSE400BSI camera placed at $1 \mathrm{~m}$ behind the sample and (b) the PIMTE camera placed close to the sample $(25 \mathrm{~cm})$. Both images correspond to the accumulation of 100 images of $200 \mathrm{~ms}$.

The beam was focalized into the CCD position and its divergence is few mrad, these explain why the diffracted spots are much wider and more separated for the sCMOS than for the CCD image. To compare these images, the spatial dimensions are transposing to the scattering vector $\mathrm{q}(q=4 \pi \sin (\theta) / \lambda$, where $\theta$ is the angle between the direct beam and the detector axes and $\lambda$ is the wavelength of the beam). The superposition of the one direction integrated diffraction peaks is presented in figure 17. It shows the similar distribution with a nice superposition of the peaks position but with a scattered intensity slightly different, particularly close to the central peak (order 0 ). As the beam focalization, the aperture, the pixel size and the QE are not the same, the magnitude scattered intensity are not easy to compare directly. We plan to have a series of future experiments in the near future to obtain more experimental results about efficiency and SNR to compare to this well-known CCD BSI. 
As first conclusion, we can conclude that similar image from sCMOS could be obtained for a total acquisition time of $20 \mathrm{~s}$ compared to the $8 \mathrm{~min}$ needed to record 100 images with the actual CCD, meaning a total time saved up to a factor 24 (limited by the exposure time).

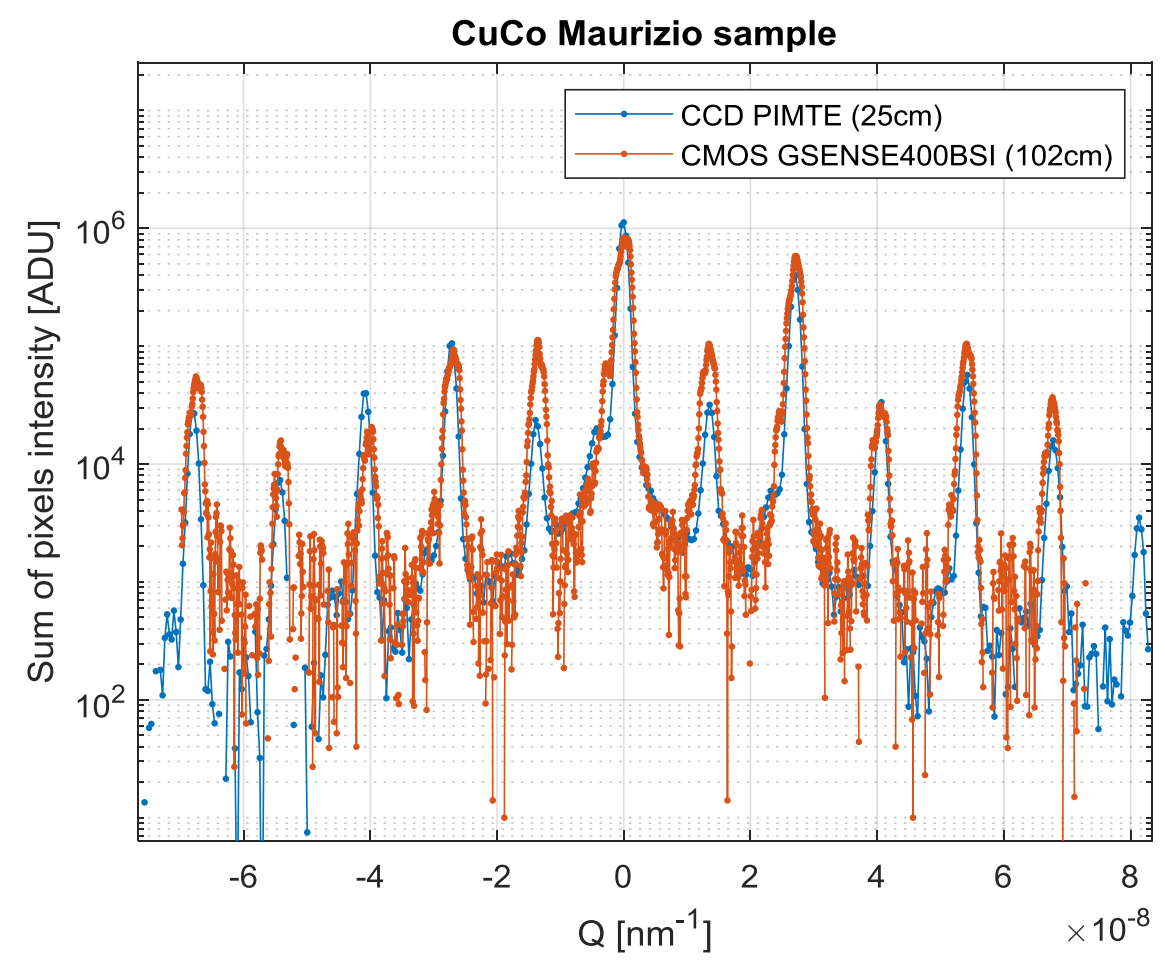

Figure 18 : Superposition and normalized the sum of the pixels in central region of the images (figure 16) obtained with CCD camera (blue line) and SCMOS camera (red line) for X-ray energy photon of $767 \mathrm{eV}$ versus the scattering vector.

This gain will change drastically the time of acquisition of an image using Fourier Transform Holography that is typically of $1 \mathrm{~h}$ nowadays to few minutes in the near future. It will be really an important gain for imaging experiment when we have to scan an external parameter (magnetic field, electric field, temperature) or even more important for time-resolved experiments (Bukin et al, 2016). The capability to acquire image with such frame rate will also dramatically expand the time window available at SEXTANTS for soft resonant magnetic X-ray Photo Correlation Spectroscopy (XPCS) only obtain recently at NSLS2 using a fast CCD (Chen et al, 2019). Lastly, it will be also a real gain for soft X-ray resonant ptychography in reflection geometry that is nowadays limited only by the coherent flux available and mechanical instabilities (Popescu et al, 2019). It is important also be noted that all these approaches will fully benefit, in terms of coherent flux and smaller beam size (using standard optics), from the new storage ring under development.

\subsection{Soft X-ray ptychography reconstruction}


In HERMES, a soft X-ray beamline dedicated for X-ray microscopy and equipped for Scanning Transmission X-ray Microscopy (STXM) at SOLEIL, a new dedicated set up for soft X-ray ptychography has been recently implemented to improve the spatial resolution capability of the beamline by replacing the 1D detector (scintillator photomultiplier tube, PMT) by the sCMOS GSENSE400BSI camera. So, the classical 2D sample scanning experiment consisting to measure the transmitted intensity is now consisting to measure the transmitted diffraction pattern recorded with the sCMOS camera.

In order to illustrate the capability of this new set-up, ptychography experiment was performed on a siemens star at $700 \mathrm{eV}$. During the experiment, diffraction patterns were collected by illuminating the sample at $? \times$ ? positions on a rectangular grid with a grid spacing of ? nm. Thanks to the fast readout of the camera and its sensitivity, the experiment was performed in less than $10 \mathrm{~min}$.

The figure 18 presented in (a) the initial image obtain using the 1D PMT detector, in (b) the ptychography reconstruction image of the part of Siemens star obtained from a series of typical speckle pattern shown in (c). The ptychography reconstruction was carried out by

using SHARP [Marchesini et al., 2016].
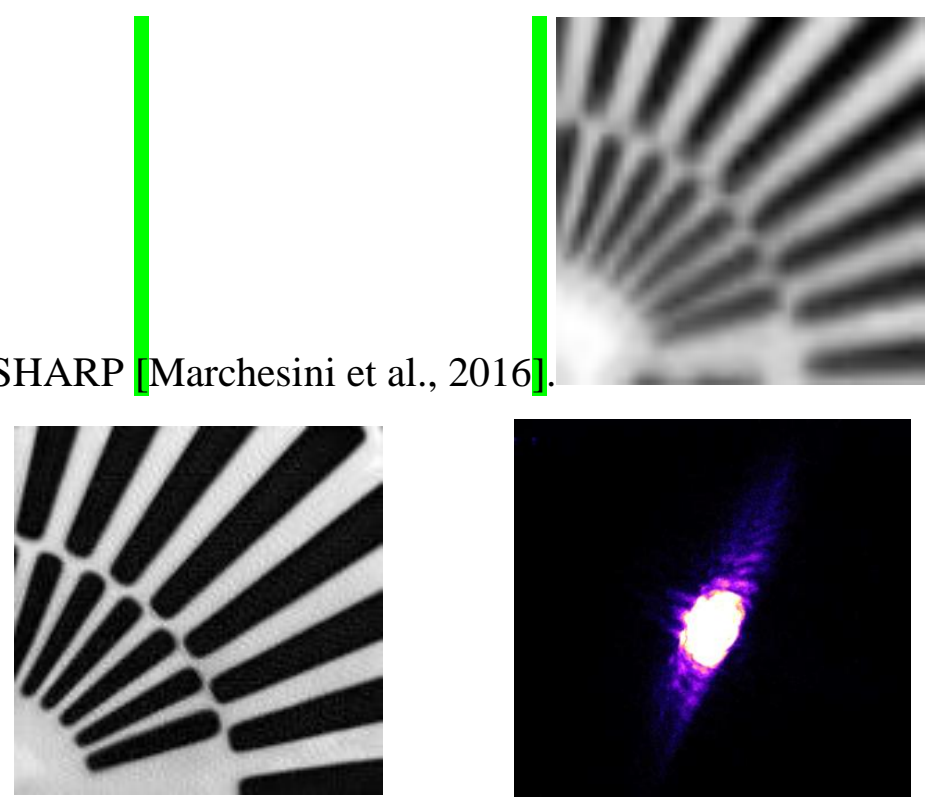

(a)

(b)

(c)

Figure 19 : (a) STXM image with beam size spot of XXXXX and (b) Ptychography reconstruction of the Siemens star. (c) Example of a speckle pattern.

To confirm these nice performances obtained on easy construct able structure, several measured have been performed, the result obtained from magnetotatic bacteria (magnetosomes) is reported here. The images have been acquired using X-ray at $700 \mathrm{eV}$, the scan step is $20 \mathrm{~nm}$. A region-of-interest (1024 px by 1024 px) has been selected on the 
sCMOS which allows to increase the frame rate up to $44 \mathrm{~Hz}$ (HRD mode) and so reduced the total acquisition to $10 \mathrm{mn}$. Again we presented, in the Figure 19.a, the lower resolution STXM classical image obtained with PMT while in the Figure. 19.b, we showed the reconstructed ptychography image performed with the ePIE method implemented in a MATLAB script developed by the NANOSCOPIUM beamline [Medjoubi et al, 2018] and used by several SOLEIL beamlines. This reconstruction allows to clearly observe the bacteria (diameter of ? $\mathrm{nm}$ ) with a very small space between two adjacent magnetosomes.

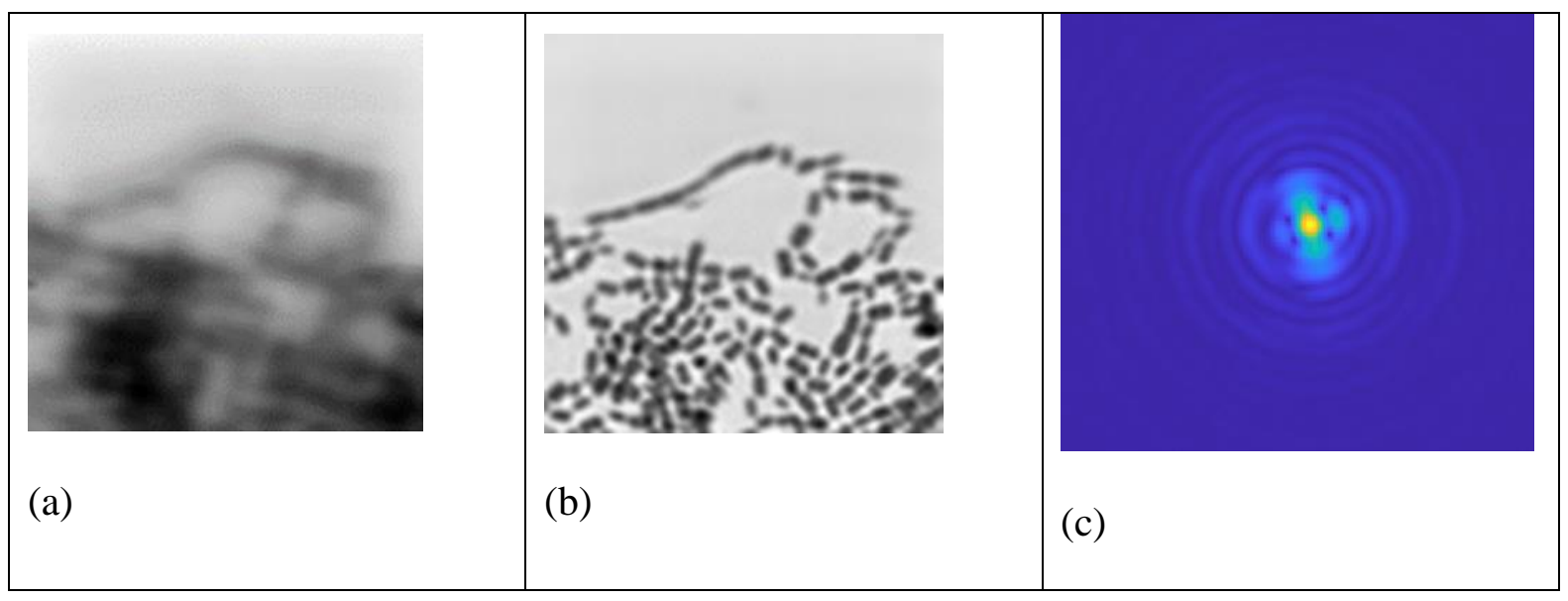

Figure 20 : (a) Classical STXM image of magnetosomes at $706 \mathrm{eV}$. (b) Ptychography reconstruction of magnetosome. (c) Example of a speckle pattern 


\section{Summary and perspectives}

A new camera based on the first world large production Back Side Illuminated scientific CMOS sensor GSENSE400BSI was developed and evaluated for soft X-ray application on several beamlines at SOLEIL synchrotron. The quantum efficiency is found to be sufficient for large field of application in the soft X-ray domain (QE $>50 \%$ for $\mathrm{E}>400 \mathrm{eV})$ and the new no-ARC (antireflective layer) has already demonstrated a high quantum efficiency of > $90 \%$ in the lower energy range and more importantly over the water window region. The readout noise $(<2$ e- rms) and the full well capacity provide a good X-ray dynamical range and an interesting single photon detection capability without decrease of the readout speed performance. This sensor has a frame rate up to $48 \mathrm{~Hz}$ (on the standard mode) that allows to dramatically reducing the acquisition time for imaging application compared to the classical camera commonly used. The new version of acquisition board allows to upgrade the performance with a lower spatial noise with DSNU < $0.2 \mathrm{e}$ - and a PRNU of $0.3 \%$. The soft Xray spatial and energy resolution has been confirmed with a limitation for low energy due to the low dead thickness and the thin Si-Epi layers.

As for similar synchrotron direct X-ray detector, the radiation hardness is a big challenge to keep good performances in the time with the high density of flux considered. The limit of dose of $200 \mathrm{~Gy}(20 \mathrm{kRad})$ and the corresponding fluence has been measured that will allow scientists to adapt the X-ray experimental conditions set-up in order to avoid damage of the sensor. A compact, cooled vacuum compatible camera prototype has been developed to be easily installed in the experimental end-stations of SEXTANTS and HERMES beamlines at SOLEIL. A first soft X-ray Resonant Scattering (REXS) acquisition from nanostructured multilayer sample has been performed on SEXTANTS beamline showing a good dynamical range with a total time saver of a factor 24 compare to their usual detector readout. A fully functional soft X-ray ptychography set-up based on this sCMOS camera has been used on the STXM end station at the HERMES beamline. Its performances and frame rate have been used to perform several reconstructions with spatial resolution down to $\sim 6 \mathrm{~nm}$.

Lastly, a new GSENSE400BSI sensor version combining a thicker Si-Epi with a high efficient process has been very recently tested by Harada team (Harada et al, 2020). It assures a very good efficiency (> $80 \%$ ) over a very large soft X-ray range and it will soon be massproduced by GPIXEL®. This new sensor will be very soon implemented on the second sCMOS HERMES camera to increase the set-up performances and particularly for the experiment at the so-called water window's carbon K-edge. Also, one other camera would be 
installed soon in the SEXTANTS COMET end-station in order to optimize the performance of acquisition time for magnetic imaging using Fourier Transform Holography.

Based on this first results and the scientist's community interest for this cost-efficient solution, a Canadian company specialist on high speed camera solution, Axis photonique [https://www.axis-photon.com] has started to produce this new soft X-ray sCMOS detector based on the SOLEIL prototype. Furthermore, others potential domains could be explored: another synchrotron beamline application, as tender X-ray SAXS (>1.2 keV) in SIRIUS beamline at SOLEIL, or for lower soft X-ray photon source based on femtosecond laser or for soft X-ray free electron laser (XFEL). In particular a test of our camera is already planned to take place in the next few months at FERMI.

To conclude, the first Back Illuminated sCMOS implementation reported in this paper could be considered as the beginning of a long series of new detector based on this technology.

Acknowledgments

The authors wish to acknowledge Hao Yuan and Adam Hitchcock of the Department of Chemistry and Biology McMaster University (Ontario, Canada) for providing us the greath helpful with Pytochography reconstruction

We also would like to acknowledge Ye(Evan) Tian from TUCSEN and Xinyang Wang from GPIXEL for the great help sharing all needed information for this work. Finally, we are very grateful to Zhang Jiang of Argonne National Laboratory for sharing the Matlab toolbox on Xray refraction of matter [Jiang 2020].

\section{References}

1. Förster, A., Brandstetter, S. \& Schulze-Briese, C. (2019). Philos. Trans. R. Soc. A Math. Phys. Eng. Sci. 377, 20180241.

2. Lambert, G., Vodungbo, B., Gautier, J., Mahieu, B., Malka, V., Sebban, S., Zeitoun, P., Luning, J., Perron, J., Andreev, A., Stremoukhov, S., Ardana-Lamas, F., Dax, A., Hauri, C. P., Sardinha, A. \& Fajardo, M. (2015). Nat. Commun. 6, 6-11.

3. Pedersoli, E., Capotondi, F., Cocco, D., Zangrando, M., Kaulich, B., Menk, R. H., Locatelli, A., Mentes, T. O., Spezzani, C., Sandrin, G., Bacescu, D. M., Kiskinova, 
M., Bajt, S., Barthelmess, M., Barty, A., Schulz, J., Gumprecht, L., Chapman, H. N., Nelson, A. J., Frank, M., Pivovaroff, M. J., Woods, B. W., Bogan, M. J. \& Hajdu, J. (2011). Rev. Sci. Instrum. 82, 043711.

4. Chauleau, J. Y., Legrand, W., Reyren, N., Maccariello, D., Collin, S., Popescu, H., Bouzehouane, K., Cros, V., Jaouen, N. \& Fert, A. (2018). Phys. Rev. Lett. 120, 37202.

5. Sacchi, M., Jaouen, N., Popescu, H., Gaudemer, R., Tonnerre, J. M., Chiuzbaian, S. G., Hague, C. F., Delmotte, A., Dubuisson, J. M., Cauchon, G., Lagarde, B. \& Polack, F. (2013). J. Phys. Conf. Ser. 425, 0-4.

6. Popescu, H., Perron, J., Pilette, B., Vacheresse, R., Pinty, V., Gaudemer, R., Sacchi, M., Delaunay, R., Fortuna, F., Medjoubi, K., Desjardins, K., Luning, J. \& Jaouen, N. (2019). J. Synchrotron Radiat. 26, 280-290.

7. Denes, P., Doering, D., Padmore, H. A., Walder, J. P. \& Weizeorick, J. (2009). Rev. Sci. Instrum. 80, 083302.

8. Jungmann-Smith, J. H., Bergamaschi, A., Brückner, M., Cartier, S., Dinapoli, R., Greiffenberg, D., Huthwelker, T., Maliakal, D., Mayilyan, D., Medjoubi, K., Mezza, D., Mozzanica, A., Ramilli, M., Ruder, C., Schädler, L., Schmitt, B., Shi, X. \& Tinti, G. (2016). J. Synchrotron Radiat. 23, 385-394.

9. Wernecke, J., Gollwitzer, C., Müller, P. \& Krumrey, M. (2014). J. Synchrotron Radiat. 21, 529-536.

10. Strüder, L. (2016). High Speed Imaging and Spectroscopy with Low Energy X-Rays. In: Jaeschke, E. J., Khan, S., Schneider, J. R. \& Hastings, J. B. (2016). Synchrotron light sources and free-electron lasers. Springer, Cham

11. Arai, Y., Miyoshi, T., Unno, Y., Tsuboyama, T., Terada, S., Ikegami, Y., Kohriki, T., Tauchi, K., Ikemoto, Y., Ichimiya, R., Ikeda, H., Hara, K., Miyake, H., Kochiyama, M., Sega, T., Hanagaki, K., Hirose, M., Hatsui, T., Kudo, T., Hirono, T., Yabashi, M., Furukawa, Y., Varner, G., Cooney, M., Hoedlmoser, H., Kennedy, J., Sahoo, H., Battaglia, M., Denes, P., Vu, C., Contarato, D., Giubilato, P., Glesener, L., Yarema, R., Lipton, R., Deptuch, G., Trimpl, M., Ohno, M., Fukuda, K., Komatsubara, H., Ida, J., Okihara, M., Hayashi, H., Kawai, Y. \& Ohtomo, A. (2010). Nucl. Instruments Methods Phys. Res. Sect. A Accel. Spectrometers, Detect. Assoc. Equip. 623, 186188.

12. Porro, M., Andricek, L., Aschauer, S., Bayer, M., Becker, J., Bombelli, L., Castoldi, A., De Vita, G., Diehl, I., Erdinger, F., Facchinetti, S., Fiorini, C., Fischer, P., Gerlach, T., Graafsma, H., Guazzoni, C., Hansen, K., Kalavakuru, P., Klär, H., Kugel, A., Lechner, P., Lemke, M., Lutz, G., Manghisoni, M., Mezza, D., Müntefering, D., Pietsch, U., Quartieri, E., Randall, M., Re, V., Reckleben, C., Sandow, C., Soldat, J., Strüder, L., Szymanski, J., Weidenspointner, G. \& Wunderer, C. B. (2012). IEEE Trans. Nucl. Sci. 59, 3339-3351.

13. Wunderer, C. B., Marras, A., Bayer, M., Glaser, L., Göttlicher, P., Lange, S., Pithan, F., Scholz, F., Seltmann, J., Shevyakov, I., Smoljanin, S., Viefhaus, J., Viti, M., Xia, Q., Zimmer, M., Klumpp, S., Gasiorek, P., Guerrini, N., Marsh, B., Sedgwick, I., Turchetta, R., Cautero, G., Farina, S., Giuressi, D., Menk, R., Stebel, L., Yousef, H., Marchal, J., Nicholls, T., Tartoni, N. \& Graafsma, H. (2014). J. Instrum. 9, C03056. 14. El Bitar, Z., Heymes, J., Kachel, M., Baudot, J., Hu-Guo, C. \& Winter, M. (2019). International Conference on Advances in Biomedical Engineering, ICABME, Vols. 2019-Octob, pp. 1-4. IEEE. 
15. Ma, C., Liu, Y., Li, J., Zhou, Q., Chang, Y. \& Wang, X. (2015). Image Sensors and Imaging Systems 2015, 9403, Proc. SPIE 9403940305.

16. Desjardins, K., Popescu, H., Mercère, P., Menneglier, C., Gaudemer, R., Thånell, K. \& Jaouen, N. (2019). AIP Conference Proceedings, 2054, 060066.

17. Wang, W. X., Ling, Z. X., Zhang, C., Jia, Z. Q., Wang, X. Y., Wu, Q., Yuan, W. M. \& Zhang, S. N. (2019). J. Instrum. 14, P02025.

18. Idir, M., Mercere, P., Moreno, T. \& Delmotte, A. (2006). Synchrotron Radiat. News. 19, 18-23.

19. Belkhou, R., Stanescu, S., Swaraj, S., Besson, A., Ledoux, M., Hajlaoui, M. \& Dalle, D. (2015). J. Synchrotron Radiat. 22, 968-979.

20. Wang, Y., Zhao, L., Hu, Z., Wang, Y., Zhao, Z., Li, L. \& Huang, Z. L. (2017). Cytom. Part A. 91, 1175-1183.

21. Janesick, J. R.(2007). Photon transfer: DN [lambda]. SPIE Press Book

22. Stackexchange https://physics.stackexchange.com/questions/15923

23. Khromova, A., Cautero, G., Giuressi, D., Menk, R., Pinaroli, G., Stebel, L., Correa, J., Marras, A., Wunderer, C. B., Lange, S., Tennert, M., Niemann, M., Hirsemann, H., Smoljanin, S., Reza, S., Graafsma, H., Göttlicher, P., Shevyakov, I., Supra, J., Xia, Q., Zimmer, M., Guerrini, N., Marsh, B., Sedgwick, I., Nicholls, T., Turchetta, R., Pedersen, U., Tartoni, N., Hyun, H. J., Kim, K. S., Rah, S. Y., Hoenk, M. E., Jewell, A. D., Jones, T. J. \& Nikzad, S. (2016). Journal of Instrumentation, 11, C11020.

24. Deiries, S., Lizon, J. L. \& Iwert, O. (2016). Adv. Opt. Mech. Technol. Telesc. Instrum. II. 9912, 99125Y.

25. Harada, T., Teranishi, N., Watanabe, T., Zhou, Q., Yang, X., Bogaerts, J. \& Wang, X. (2019). Appl. Phys. Express. 12, 082012.

26. Lumb D.H., Hopkinson R., W. A. A. (1984). Nucl. Inst. Methods Phys. Res. 221, $150-158$.

27. Janesick, J. R.(2001). Scientific Charge-Coupled Devices. SPIE Press Book

28. Ravotti, F. (2018). IEEE Trans. Nucl. Sci. 65, 1440-1464.

29. Clarke, R. (1994). Nucl. Inst. Methods Phys. Res. A. 347, 529-533.

30. Hancock, B. R. \& Soli, G. A. (1997). IEEE Trans. Nucl. Sci. 44, 1957-1964.

31. Jaouen, N., Tonnerre, J. M., Kapoujian, G., Taunier, P., Roux, J. P., Raoux, D. \& Sirotti, F. (2004). J. Synchrotron Radiat. 11, 353-357.

32. Bukin, N., McKeever, C., Burgos-Parra, E., Keatley, P. S., Hicken, R. J., Ogrin, F. Y., Beutier, G., Dupraz, M., Popescu, H., Jaouen, N., Yakhou-Harris, F., Cavill, S. A. \& Van Der Laan, G. (2016). Sci. Rep. 6, 1-10.

33. Chen, X. M., Farmer, B., Woods, J. S., Dhuey, S., Hu, W., Mazzoli, C., Wilkins, S. B., Chopdekar, R. V., Scholl, A., Robinson, I. K., De Long, L. E., Roy, S. \& Hastings, J. T. (2019). Phys. Rev. Lett. 123, 1-6.

34. Medjoubi, K., Baranton, G. \& Somogyi, A. (2018). Microsc. Microanal. 24, 252-253.

35. Harada, T., Teranishi, N., Watanabe, T., Zhou, Q., Bogaerts, J. \& Wang, X. (2020). Appl. Phys. Express. 13, 016502

36. Burns, P. (2009). SFRmat3 Script (http://losburns.com), MATLAB script.

37. Jiang, Z (2020). Toolbox: X-ray Refraction of Matter (https://www.mathworks.com/matlabcentral/fileexchange/6026-toolbox-X-rayrefraction-of-matter), MATLAB Central File Exchange. 